\title{
Augmented Vessels for Quantitative Analysis of Vascular Abnormalities and Endovascular Treatment Planning
}

\author{
Wilbur C. K. Wong* and Albert C. S. Chung
}

\begin{abstract}
Endovascular treatment plays an important role in the minimally invasive treatment of patients with vascular diseases, a major cause of morbidity and mortality worldwide. Given a segmentation of an angiography, quantitative analysis of abnormal structures can aid radiologists in choosing appropriate treatments and apparatuses. However, effective quantitation is only attainable if the abnormalities are identified from the vasculature. To achieve this, a novel method is developed, which works on the simpler shape of normal vessels to identify different vascular abnormalities (viz. stenotic atherosclerotic plaque, and saccular and fusiform aneurysmal lumens) in an indirect fashion, instead of directly manipulating the complex-shaped abnormalities. The proposed method has been tested on three synthetic and 17 clinical data sets. Comparisons with two related works are also conducted. Experimental results show that our method can produce satisfactory identification of the abnormalities and approximations of the ideal post-treatment vessel lumens. In addition, it can help increase the repeatability of the measurement of clinical parameters significantly.
\end{abstract}

Index Terms-Angiography, augmented vessel, endovascular treatment planning, quantitative analysis, vessel tracking.

\section{INTRODUCTION}

$\mathbf{E}$ NDOVASCULAR treatment is a therapy performed inside vessels with the assistance of two-dimensional (2-D) angiography and micro-catheters. It plays an important role in minimally invasive treatments of patients with vascular diseases [1]. Vascular disease is one of the major causes of morbidity and mortality worldwide, particularly cerebrovascular diseases, such as intracranial aneurysms, carotid stenoses and arteriovenous malformations. In a clinical review [2], it was found that approximately $3.6 \%-6 \%$ of the general population are suffering from intracranial aneurysms. As such, diagnostic imaging scientists are seeking new technologies aimed at providing a more reliable diagnostic evaluation for endovascular treatments and therapeutic assessment of vascular diseases with three-dimensional (3-D) angiographic information. Magnetic resonance angiography (MRA), computed tomography

Manuscript received December 17, 2005; revised February 20, 2006. This work was supported by the Hong Kong Research Grants Council (HK RGC) under Grant HKUST6209/02E and Grant 612305. Asterisk indicates corresponding author.

*W. C. K. Wong is with the Lo Kwee-Seong Medical Image Analysis Laboratory, Department of Computer Science, The Hong Kong University of Science and Technology, Kowloon, Hong Kong (e-mail: cswilbur@cs.ust.hk).

A. C. S. Chung is with the Lo Kwee-Seong Medical Image Analysis Laboratory, Department of Computer Science, The Hong Kong University of Science and Technology, Kowloon, Hong Kong (e-mail: achung@ @s.ust.hk).

Digital Object Identifier 10.1109/TMI.2006.873300 angiography (CTA), and 3-D rotational angiography (RA) are examples of these technologies.

Vascular segmentation of 3-D angiographies can provide patient-specific 3-D vascular models. This is very useful in endovascular treatment planning [3]. Nevertheless, even if a vascular model is obtained, measuring the clinical parameters of interest still remains a problem. In particular, measuring the neck width and dome height of a cerebral aneurysm ${ }^{1}$ are the most difficult tasks. These measurements, however, would be easier to take if the aneurysmal sac is demarcated from its parent arteries. Interactive or automatic quantitative analyzes on the lesion would then become feasible, because the pathological structures could be processed exclusively. The estimation of Guglielmi detachable coil's (GDC) size and shape could be obtained from simple morphological operations (opening and closing) or complex algorithms like deformable models [6] that are applied to the detached sac volume. In the case of arterial coarctation, if the volume of atherosclerotic plaque that causes the stenosis is estimated, a best-fitting stent for an endovascular recanalization can then be deduced from the plaque dimensions.

The identification of abnormal vascular structures would also help angle selection for optimal working projections. ${ }^{2}$ With the knowledge of the shape and orientation of an aneurysmal neck, the method proposed by van der Weide et al. [8], which is based upon minimizing the area of the aneurysmal neck in projected images, could be used for the angle selection. Once the aneurysmal lumen is demarcated, another more advanced choice would be the one developed by Wilson et al. [9] in which the optimal working angle is determined via a minimization of the area of overlaps between adjoining vessels and the sac. Similar techniques could be developed for selecting the optimal working angle in an endovascular recanalization if the volume of atherosclerotic plaque is estimated.

Nonetheless, little work has been done on the identification of abnormal vascular structures in angiographies. In the literature, only a few authors have suggested methods to detach aneurysmal lumens from vasculatures. Wilson et al. [9] suggested using the distance map of an aneurysmal sac center to determine the aneurysmal volume. They identified and excluded high-intensity regions in the distance map, and

\footnotetext{
${ }^{1}$ An aneurysm is a localized abnormal dilation of a blood vessel. The neck width and the dome height of a cerebral aneurysm are important information for selecting proper apparatus (such as micro-balloons [4]) and can help predict immediate outcomes of an occlusion with Guglielmi detachable coils (GDC) [5].

${ }^{2}$ The aim of optimal working angle selection is to seek a clear view of a stenotic lumen or an aneurysmal sac and its neck so as to avoid foreshortening of the lesions and overlapping with other objects in their 2-D angiograms [7]-[9].
} 
inpainted the resultant empty regions by interpolation between neighboring low-intensity regions. Then all voxels that lie within the distances in the inpainted map from the sac center are classified into the aneurysmal volume. McLaughlin and Noble [10] determined aneurysmal voxels as the voxels that are closest to an aneurysmal mesh identified by a user with the seed and cull algorithm. These two algorithms, however, have difficulty giving satisfactory results on wide-neck ${ }^{3}$ saccular aneurysms and fusiform aneurysms, as is shown in Section IV.

In this paper, we develop a novel approach to identifying a variety of vascular abnormalities, viz. stenotic atherosclerotic plaque, and saccular and fusiform aneurysmal lumens from vasculatures on a unified framework. We take a very different approach as compared with other published methods. Instead of manipulating the pathological structures with sophisticated algorithms, we identify the abnormalities by explicitly modeling their opposite, i.e., normal vessels. As compared with the complex shape of the abnormal lumens, our method works on the relatively simpler shape of normal vessels. The abnormal vascular structures are then determined as the complement of the approximated normal vessels. For instance, an aneurysmal lumen is seen as the surplus volume in the vasculature in respect to the normal vessel model, whereas the absent volume in a stenotic lumen is an estimate of the atherosclerotic plaque volume.

We refer to this normal vessel model as the "augmented vessel." The term "augmented" is borrowed from the terminology "augmented reality," which refers to the technology that integrates computer-generated objects into real-world environments [11]. Our augmented vessels are computer-generated vessels aimed at estimating a portion of post-treatment vessel lumens under conditions that either 1) a stent successfully restores the width of a stenotic lumen which is comparable to the widths of normal lumen segments that are proximal and distal to the coarctation, 2) an aneurysmal sac is completely packed with GDC, or 3) an aneurysmal lumen is occluded perfectly by stent grafts [12]. Those conditions are regarded as clinically ideal, since the post-treatment vessel lumens approximated are very similar to normal lumens. It is perceived that such approximation does not take hemodynamics of vasculature into account. We are neither aiming at producing accurate modelings of post-treatment lumens that are hemodynamically stable nor approximating actual lumens after endovascular treatments. Instead, our goal is to identify the volumes of abnormal vascular structures (based on normal counterparts) so as to ease measurement of clinical parameters, make the measurements more consistent yet less subjective and, as a result, allow endovascular treatment planning to be facilitated.

The rest of the paper is organized as follows. The proposed method is described in Section II, followed by several implementation issues in Section III. Experimental results on synthetic and clinical data sets are presented in Section IV. An in vivo study and its findings are given in Section V. Finally, dis-

\footnotetext{
${ }^{3}$ Sanders et al. suggested using a sac-to-neck ratio to access the success rate of a Guglielmi detachable coil (GDC) occlusion of an aneurysm [4]. This ratio is defined as the diameter of an aneurysmal sac divided by the diameter of the sac opening. An aneurysm with a sac-to-neck ratio of 2.0 or more is optimal for GDC occlusion, whereas a sac-to-neck ratio nearing 1.0 suggested that the lesion is a wide-neck aneurysm which is more difficult or may be impossible to treat with GDC occlusion.
}

cussions and the conclusion are presented in Sections VI and VII, respectively.

\section{DESCRIPTION OF THE METHOD}

For the purpose of this study, we assume that a topologically and morphologically correct vascular segmentation (i.e., with no holes and cavities) is available. Without loss of generality, the segmentation is represented in a binary image volume with voxel label equals one for the vessel lumen class and zero for the background class. Throughout this paper, augmented vessels are constructed with reference to this vascular segmentation.

In this section, we begin by describing the construction of augmented vessels, which consists of two major phases: 1) determination of vessel widths and centerlines (Section II-A); 2) production of an explicit vascular surface model (Section II-B). We then present demarcation of aneurysmal sacs and volume estimation of stenosis atherosclerotic plaque in terms of a simple set operation in Section II-C.

\section{A. Vessel Widths and Centerlines}

In this paper, we focus on images that contain arteries. In order to model all possible vasculatures found in clinical data sets, a generic-enough model is required such that the model can be molded into the imaged vessels ranging from simple lumen segments to complex n-way branching junctions. As such, we employ the most intuitive and commonly used vessel model found in the literature-circular cross-sectional tube [13]-[18] - to initiate the construction of augmented vessels: a tube is used to model a single lumen segment; and multiple tubes are required in the case of an n-way branching vascular junction.

1) Determination of Vessel Widths and Centerline End-Points: In this simple initialization model, the widths ( $2 \times$ radius of local circular cross sections) of a tube and the location of the tube centerline are the only attributes subject to change. A user is requested to select two points in a 3-D space to define the end-points per tube centerline which is used to initiate the construction of augmented vessels. They should be selected at disease-free portions of vessels of interest such that the tube centerline either passes by a saccular aneurysmal lumen or passes through a fusiform aneurysmal or a stenotic lumens. The circles in Fig. 1 are possible centerline end-points selected by a user in disease-free regions of two aneurysmal cases and a stenotic case.

The widths of the tube along the centerline are then determined by using the linear interpolation between the local vessel widths at the two selected points. The reason for imposing such a hard constraint on the tube widths is that we need a strong shape prior to restrict the morphology of the augmented vessels in a pathological region. Otherwise, we may have an overfit initialization model that is molded to a complicated pathological lumen (e.g., a poststenotic dilated lumen [19], an asymmetrically dilated aneurysmal lumen and a coarctation), if the tube widths are allowed to vary out of the range bounded by the end-points' local vessel widths.

2) Representation of Vessel Centerline: The tube centerline is modeled using an open parametric zero-tension cardinal spline. Compared with the conventional open B-splines [9], 


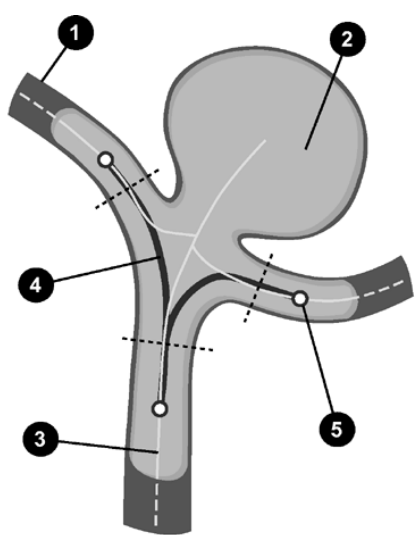

(a)

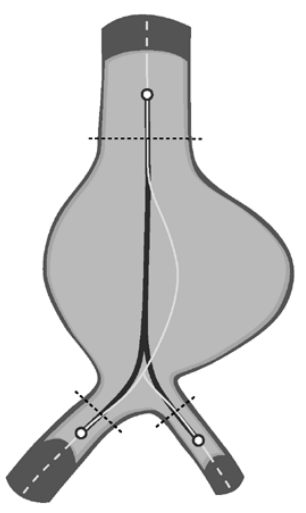

(b)

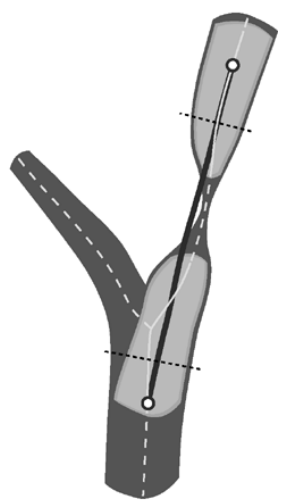

(c)
Fig. 1. Possible end-points selected by a user, their corresponding estimated centerlines and ridges in Euclidean distance (ED) maps of (a) a saccular aneurysmal lumen, (b) a fusiform aneurysmal lumen and (c) a stenotic lumen. The portion toward the far end of the lumen from the dotted straight line denotes the disease-free region. The components are labeled: 1, a blood vessel wall; 2, an arterial lumen; 3, a ridge in an ED map; 4, an estimated centerline; 5 , a user selected end-point. Also, notice the differences between the estimated centerlines and the ridges in the ED map.

[13], cardinal splines have the advantage of being able to pass through the knots, which makes the representation more intuitive in subsequent approximation of the vessel centerlines. A cardinal spline fragment between the $i$ th and $(i+1)$ th knots is defined as follows:

$\mathbf{C}_{i, i+1}(u)=\left[\begin{array}{c}(u-i)^{3} \\ (u-i)^{2} \\ (u-i) \\ 1\end{array}\right]^{\mathrm{T}}\left[\begin{array}{cccc}2 & -2 & 1 & 1 \\ -3 & 3 & -2 & -1 \\ 0 & 0 & 1 & 0 \\ 1 & 0 & 0 & 0\end{array}\right]\left[\begin{array}{c}\mathbf{P}_{i} \\ \mathbf{P}_{i+1} \\ \mathbf{P}^{\prime}{ }_{i} \\ \mathbf{P}^{\prime}{ }_{i+1}\end{array}\right]$

$$
\mathbf{P}_{j}^{\prime}=(1-s)\left(\mathbf{P}_{\min (j+1, N-1)}-\mathbf{P}_{\max (0, j-1)}\right)
$$

where $u \in \mathbb{R}, i \leq u \leq i+1$ is a parametric variable. $\mathbf{P}_{i}$ and $\mathbf{P}_{i+1}$ denote the $i$ th and $(i+1)$ th knots, respectively. $N$ is the total number of knots. The notation $\mathbf{P}^{\prime}{ }_{j}$ denotes the tangent at point $\mathbf{P}_{j}$ and is approximated using (2). In this paper, we set $s=$ 0 to ensure that the spline fragments are joined smoothly at each corresponding knot. The complete cardinal spline composed by the individual fragments is, therefore, described as

$$
\mathbf{C}_{u}=\mathbf{C}_{j, j+1}(u), \quad \text { for } j \leq u \leq j+1
$$

where $j \in \mathbb{N}, 0 \leq j \leq N-2$ and $0 \leq u \leq N-1$.
3) Approximation of Vessel Centerline: The approximation of the vessel centerline is treated as a spline registration problem under a fixed end boundary condition [20]. We solve the registration problem using the active contour models, Snakes [21]. Our cardinal splines have two types of parameters, intrinsic and extrinsic parameters. The intrinsic parameters include the model constraint functionals and image functional, whereas the extrinsic parameters define the search space of the registration.

The model constraint functionals are length penalty, tensile and flexural strengths. The length penalty acts as a soft constraint to avoid redundant tortuousness. The tensile and flexural strengths control the resistance of the spline to stretching and bending deformations, respectively. The image functional, primarily for anatomy penalty, poses a strong constraint on the registration such that the tube centerline at the final equilibrium state follows the trajectory of vessels of interest. Unlike the commonly used image functionals, such as those based on intensity gradient magnitude [13], [16]-[18], [22], [23], ours is distinctive. It takes volumetric information into account, instead of lumen boundaries, to encourage large volumetric overlap between the tube and the vascular structures. This allows the estimated centerline outside the confines of a narrowed lumen which can help identification of stenotic asymmetry (a useful information in a clinical diagnosis [24]), as depicted in Fig. 1(c).

Our energy functional to be minimized is defined as follows:

$$
E^{\mathbf{C}}=\underbrace{\gamma_{\mathrm{t}}^{\mathbf{C}} E_{\text {tensile }}^{\mathbf{C}}+\gamma_{\mathrm{f}}^{\mathbf{C}} E_{\text {flexural }}^{\mathbf{C}}+\gamma_{1}^{\mathbf{C}} E_{\text {length }}^{\mathbf{C}}}_{\text {model constraint functionals }}+\underbrace{\gamma_{\mathrm{a}}^{\mathbf{C}} E_{\text {anatomy }}^{\mathbf{C}}}_{\text {image functional }}
$$

where

$$
\begin{aligned}
E_{\text {tensile }}^{\mathbf{C}} & =\int_{\mathbf{C}}\left|\mathbf{C}_{u}\right|^{2} d u \\
E_{\text {flexural }}^{\mathbf{C}} & =\int_{\mathbf{C}}\left|\mathbf{C}_{u u}\right|^{2} d u \\
E_{\text {length }}^{\mathbf{C}} & =\int_{\mathbf{C}}\left|\mathbf{C}_{u}\right| d u \\
E_{\text {anatomy }}^{\mathbf{C}} & =\int_{\mathbf{C}} 1-\mathcal{V}(\mathbf{C}) d u .
\end{aligned}
$$

The weights $\gamma_{.}^{\mathbf{C}}$ control the inferences of the individual energy functionals $E^{\stackrel{\mathbf{C}}{*}}, \mathbf{C}$ is the abbreviation for the spline $\mathbf{C}(u), \mid$. $\mid$ is the $l^{2}$-norm operator and $\mathcal{V}(\mathbf{C}) \in[0,1]$ denotes a scalar function which returns the areas of the circular cross section of the tube at $\mathbf{C}(u)$ that are occupied by the vascular structures in the percentage. The solid lines labeled as 4 in Fig. 1 are possible vessel centerlines estimated using our method.

The search space of this minimization problem is the Cartesian coordinates of all movable knots (i.e., all the knots, except $\mathbf{P}_{0}$ and $\left.\mathbf{P}_{N-1}\right)$. Unlike the works done by Wilson et al. [9] and Frangi et al. [13], we do not assume the number of knots (also known as order) in the spline is fixed (i.e., single-order). A multi-order (MO) approach similar to the multi-resolution B-spline mesh illustrated in [25] for free-form MRI registration is adopted. The order of cardinal splines is automatically determined in a coarse to fine fashion. Instead of doubling the 
number of knots at the next higher order (finer) level as proposed in [25], we increase one knot at each higher level. Therefore, comparing with the previous works (i.e., [9] and [13]) done, our approach is more generic and novel. One might suggest to take the single-order approach so as not to overcomplicate the vessel centerline approximation. Later in experiments on a clinical data set, we demonstrate that such approach can easily get trapped at local optimum, especially when the order of splines is high ( $>2$ movable knots). However, if the order is low $(\leq 2$ movable knots), splines may suffer from low degree of freedom (DOF) and, hence, are unable to model portions that exhibit relatively high curvature. The implementation of this minimization procedure is presented in Section III-B.

\section{B. Explicit Vascular Surface Model}

To construct the augmented vessels, determining the widths and the centerlines of the vessels are necessary but not sufficient. We also need to define an explicit closed surface to represent the augmented vessel lumen boundaries. This explicit surface plays an important role in identification of the abnormal vascular structures.

In the demarcation of an aneurysmal lumen, for instance, the augmented vascular surface acts like a 3-D "cookie cutter" that crops the vascular structure, analogous to dough, into two parts, "cookie dough" and "wasted dough" (see Fig. 2(a) and the small figure on the lower right corner of Fig. 2(b), the partitions labeled $2 \mathrm{a}$ and $2 \mathrm{~b}$ are contributed to the "wasted dough" and the partition labeled 3 is the "cookie dough"). The "cookie dough" is an estimate of a portion of the vessel lumen in the region of interest after a perfect endovascular embolization. The "wasted dough," on the other hand, consists of the suspected aneurysmal lumen labeled $2 \mathrm{a}$ and the regions of no interest labeled $2 \mathrm{~b}$. After this partitioning, the suspected aneurysmal lumen can be easily selected in the "wasted dough."

In the case of the volume estimation of stenotic atherosclerotic plaque, the explicit surface has its intuitive physical meaning. It represents an inflated balloon catheter in balloon angioplasty, as shown in Fig. 2(c). The spaces that are not occupied by the vascular structures inside the explicit closed surface may correspond to the atherosclerotic plaque [i.e., the partitions labeled 5 in the small figure on the lower left corner of Fig. 2(c)]. The suspected plaque volume can then be identified and selected manually with ease. Thus, estimation of the atherosclerotic plaque volume becomes feasible.

1) Surface Representation and Initialization: The augmented vascular surface is represented as a vector-valued parametric function

$$
\mathbf{S}(u, v)=[x(u, v), y(u, v), z(u, v)]^{\mathrm{T}}
$$

where $u$ and $v$ are parametric variables. It is initialized as a closed surface $\mathbf{S}_{\mathbf{0}}$ that represents the augmented vessels' morphological shape (i.e., composition of circular cross-sectional tubes) explicitly. Details of the initialization are presented in Section III-C.

2) Surface Matching: Although, arteries have thicker walls that help retain the lumen boundaries more-or-less circle around the vessel centerlines [26], the cross-sectional shape can still deviate from that being perfectly circular. Hence, the circular

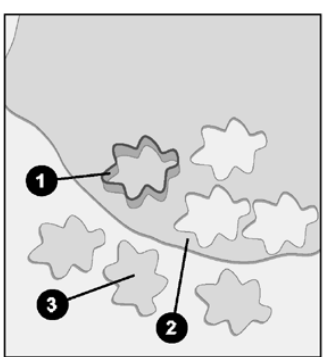

(a)

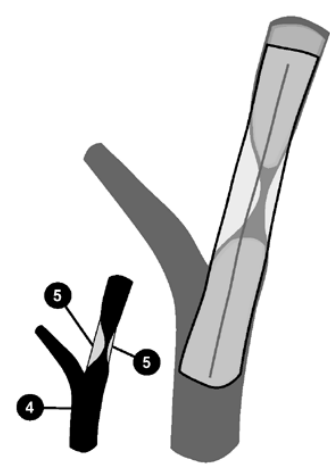

(c)

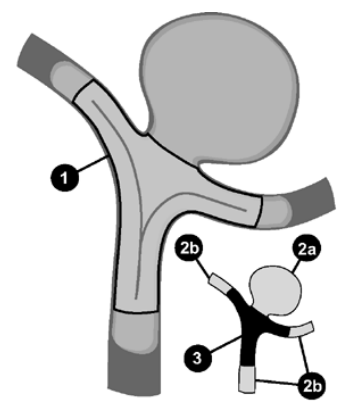

(b)

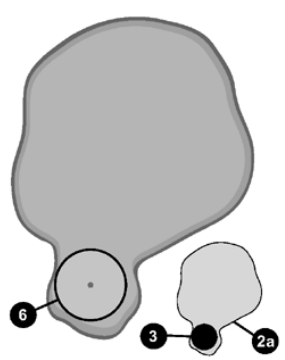

(d)
Fig. 2. Explicit vascular surface models. An analogy between cookie cutter/ doughs and explicit vascular surface model/vessel lumens in the demarcation of an aneurysmal lumen: (a) cookie cutter labeled 1, waste dough labeled 2, and cookie dough labeled 3; and (b) explicit vascular surface model labeled 1, aneurysmal lumen labeled 2a (a part of the "wasted dough"), regions of no interest labeled $2 \mathrm{~b}$ (parts of the "wasted dough") and augmented vessels labeled 3 ("cookie dough"). (c) Intuitive physical meaning of an explicit vascular surface model in the volume estimation of stenotic atherosclerotic plaque, an inflated balloon catheter in balloon angioplasty. Label 4, vascular structures in the pretreatment vascular segmentation; and label 5, approximated atherosclerotic plaque (absent vascular structures). (d) Cross-sectional shapes of an aneurysmal lumen and its parent vessel lumen. The shape of the parent vessel lumen deviates from a circular cross-sectional tube. The estimated aneurysmal lumen labeled $2 \mathrm{a}$ is overestimated if the initial surface model $\mathbf{S}_{\mathbf{0}}$ labeled 6 is used to "cut" the vascular structure.

cross-sectional tube representation is too primitive to approximate the arterial lumens in clinical data sets. As depicted in Fig. 2(d), it is very likely that the obtained aneurysmal lumen is overestimated (label 2a), if the initial surface model $\mathbf{S}_{\mathbf{0}}$ (label 6 ) is used to "cut" the vascular structure.

To avoid such overestimation, we need to make fine adjustments on $\mathbf{S}_{0}$ by matching it to the nearby lumen boundaries in the vascular segmentation. The surface matching is performed in an energy minimization based nonrigid registration framework. The augmented vascular surface $\mathbf{S}$ is a floating surface, which freely deforms (in a global sense) into a reference surface $\mathbf{M}$, the lumen boundaries in the segmentation. In order to homogenize the representation of the two surfaces (i.e., the deformation medium), the lumen boundaries are explicitly represented by the iso-surface obtained from the Marching Cubes algorithm [27].

Although the deformation is globally free-form, we do impose local constraints. These constraints dictate the evolution of $\mathbf{S}$ such that it has an affinity with the nearby lumen boundaries (boundaries of the parent vessel lumen) but not the distant counterparts (e.g., the aneurysmal and stenotic lumen boundaries). We propose two novel external energy functionals to impose those constraints. They are snap functional and anti-col- 
(a)

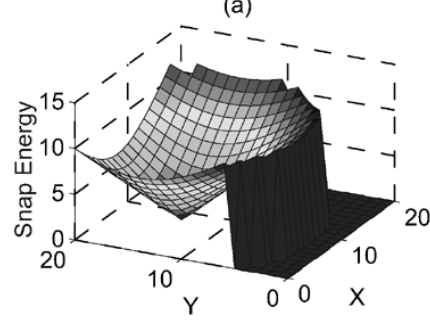

(c)

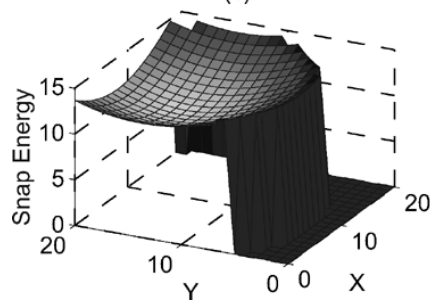

(b)

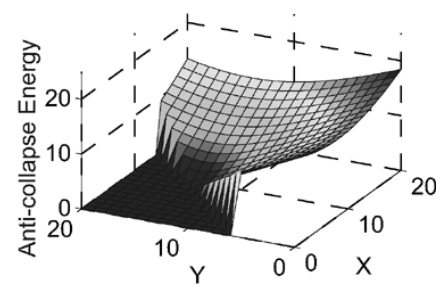

(d)

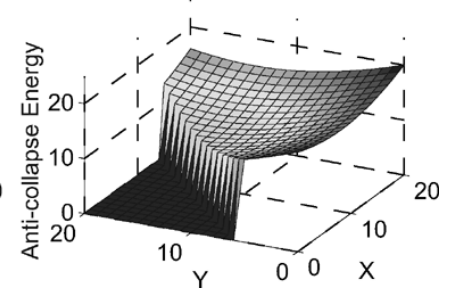

Fig. 3. Energy values of the snap and the anti-collapse functionals. Left column: the snap energy (at $z=0$ ) of a point whose nearest point on $\mathbf{M}$ is $[8,14,0]^{\mathrm{T}}$ when $R=12$ in different values of $p$, (a) $p=0.1$ and (c) $p=10$. Right column: the anti-collapse energy (at $z=0$ ) of a point whose initial position is $[8,14,0]^{\mathrm{T}}$ when $\hat{n_{0}}=[-1 / \sqrt{2}, 1 / \sqrt{2}, 0]^{\mathrm{T}}$ in different values of $r$, (b) $r=10$ and (d) $r=0.1$.

lapse functional. These two functionals are very different from the classical functionals proposed in the literature on deformation models [21], [28]-[31], such as the functionals built upon negative intensity, negative intensity gradient magnitude, intensity gradient vector and contour/surface curvature. Our snap and anti-collapse functionals are defined on geometric displacement fields of $\mathbf{S}$ to $\mathbf{M}$ and $\mathbf{S}_{\mathbf{0}}$, respectively. This allows embedding shape priors into the deformation regardless of the representation of the deformable model (see Section VI-A for the discussion on model representation). Classical tensile and flexural functionals are also employed for surface regularization.

3) Snap Functional: The snap functional favors $\mathbf{S}$ to stick to $\mathbf{M}$. This alleviates the unrealistic shape of $\mathbf{S}_{\mathbf{0}}$ and gives a better modeling of the lumen boundaries in the vascular segmentation. It is defined as

where

$$
E_{\mathrm{snap}}^{\mathrm{S}}=\int_{\mathbf{S}} \mathcal{E}_{\mathrm{snap}}^{\mathbf{S}} d u d v
$$

$\mathcal{E}_{\text {snap }}^{\mathbf{S}}=\left\{\begin{array}{ll}\operatorname{ndism}_{\mathbf{M}}(\mathbf{S})+p \times e^{\frac{-\operatorname{ndis}_{\mathbf{M}}(\mathbf{S})}{p}}, & \text { for } \operatorname{ndis} \mathbf{M}(\mathbf{S}) \leq R \\ 0, & \text { otherwise }\end{array}\right.$.

The symbol $\mathbf{S}$ in the integrand is shorthand for $\mathbf{S}(u, v)$, $n \operatorname{dism}_{M}(\mathbf{S})$ returns the shortest Euclidean distance (ED) from $\mathbf{S}(u, v)$ to $\mathbf{M}, p$ is a positive nonzero constant that controls the energy drop rate around the minimum (i.e., $\operatorname{ndis} \mathbf{M}(\mathbf{S})=0$ ). A smaller $p$ gives a more abrupt decrease in the snap energy as $\operatorname{ndis} \mathbf{M}(\mathbf{S})$ approaches zero and, hence, increases the strength of the snap constraint. $R$ is a positive constant that defines a circular snap range, outside which the energy vanishes. In other words, it controls the sensitivity of $\mathbf{S}$ toward $\mathbf{M}$ (the sensitivity is proportional to the value of $R$ ) and plays an important role in discouraging $\mathbf{S}$ to stick to the distant lumen boundaries. The left column of Fig. 3 shows the snap energy (at $z=0$ ) of a point whose nearest point on $\mathbf{M}$ is $[8,14,0]^{\mathrm{T}}$ when $R=12$ in different values of $p$.
4) Anti-Collapse Functional: Though the snap functional can avoid $\mathbf{S}$ marching toward aneurysmal lumen boundaries that are relatively far away from $\mathbf{S}_{\mathbf{0}}$, distant diseased lumen boundaries seldom exist in the cases of stenosis where lumens narrow gradually. The snap functional may drive $\mathbf{S}$ to the stenotic lumen boundaries and make the augmented vascular surface collapsed. The anti-collapse functional is, therefore, proposed as a remedy for this problem. The form of this functional is similar to the snap counterpart. Instead of favoring a deformation that minimizes the shortest geometric distances between $\mathbf{S}$ and $\mathbf{M}$, the anti-collapse functional encourages the magnitude of the negative displacement (displacement is negative if its direction is opposite to the outward surface normal) of $\mathbf{S}$ from $\mathbf{S}_{\mathbf{0}}$ to be minimized. In other words, it favors $\mathbf{S}$ to restore its initial position (i.e., maintain its prior shape) only when it is collapsed/shrunk. This functional is given as

$$
E_{\text {anti-collapse }}^{\mathrm{S}}=\int_{\mathbf{S}} \mathcal{E}_{\text {anti-collapse }}^{\mathrm{S}} d u d v
$$

where

$$
\mathcal{E}_{\text {anti-collapse }}^{\mathbf{S}}=\left\{\begin{array}{ll}
|\Delta \mathbf{S}|+\frac{1}{r} \times e^{-r \times|\Delta \mathbf{S}|}, & \text { for } \hat{n_{0}} \cdot \Delta \mathbf{S} \leq 0 \\
0, & \text { otherwise }
\end{array} .\right.
$$

The vector $\Delta \mathbf{S}$ equals $\mathbf{S}(u, v)-\mathbf{S}_{\mathbf{0}}(u, v) .|\cdot|$ is the $l^{2}$-norm operator and the positive nonzero parameter $r$ controls the rate of the initial position restoration. The vector $\hat{n_{0}}$ denotes the outward normal vector at $\mathbf{S}_{\mathbf{0}}(u, v)$. It helps define the null energy positive displacement half-space. Thus, the anti-collapse forces do not drive the surface ahead of its initial position or in other words they do not help inflate/expand the surface. The right column of Fig. 3 shows the anti-collapse energy (at $z=0$ ) of a point whose initial position is $[8,14,0]^{\mathrm{T}}$ when $\hat{n_{0}}=[-1 / \sqrt{2}, 1 / \sqrt{2}, 0]^{\mathrm{T}}$ in different values of $r$.

5) Regularization Functionals: Standard regularization functionals, tensile and flexural, are also employed. Similar to the spline registration presented in Section II-A-3, these two functionals make $\mathbf{S}$ act like a membrane and a thin-plate to avoid development of surface singularities. The tensile and flexural functionals are written as

$$
\begin{aligned}
E_{\text {tensile }}^{\mathbf{S}} & =\int_{\mathbf{S}}\left|\mathbf{S}_{u}\right|^{2}+\left|\mathbf{S}_{v}\right|^{2} d u d v \\
E_{\text {flexural }}^{\mathbf{S}} & =\int_{\mathbf{S}}\left|\mathbf{S}_{u u}\right|^{2}+2\left|\mathbf{S}_{u v}\right|^{2}+\left|\mathbf{S}_{v v}\right|^{2} d u d v .
\end{aligned}
$$

6) Optimization Procedure: The overall energy functional to be minimized is, therefore, given as follows (similar to $E^{\mathbf{C}}$ in (4)

$$
E^{\mathbf{S}}=\underbrace{\lambda_{\mathrm{s}}^{\mathbf{S}} E_{\mathrm{snap}}^{\mathbf{S}}+\lambda_{\mathrm{a}}^{\mathbf{S}} E_{\text {anti-collapse }}^{\mathbf{S}}}_{\text {external functionals }}+\underbrace{\lambda_{\mathrm{t}}^{\mathbf{S}} E_{\text {tensile }}^{\mathbf{S}}+\lambda_{\mathrm{f}}^{\mathbf{S}} E_{\text {flexural }}^{\mathbf{S}}}_{\text {regularization functionals }} .
$$

Due to the high dimensionality (dimension equals $3 n$, where $n$ is the number of sample points on $\mathbf{S}$ whose order of magnitude is at least hundreds) of $E^{\mathrm{S}}$, we take a deterministic approach to searching for a local minimum. It is a standard approach to free- 
form deformation problems [32]. The Lagrangian mechanics is applied to construct a dynamical system that is dictated by the Lagrangian $E^{\mathbf{S}}$ in (16). Hence, the equilibrium state (the matched $\mathbf{S}$ ) is obtained as the solution to the Euler-Lagrange equations. The motion equations for each sample points on $\mathbf{S}$ are then derived using the gradient-descent method. The motion equations and their discrete representations are presented in Section III-D.

\section{Identification of Abnormalities}

Once the augmented vascular surface model is constructed, it is transformed into a binary volume in the same resolution as in the pre-treatment vascular segmentation. Voxels enclosed by the surface model are labeled as ones and the background is labeled with zeros. Details of the transformation are outlined in Section III-E. In this paper, we consider the two most commonly found abnormalities in the human arterial network: aneurysm and stenosis. An aneurysm is a local abnormal dilation of a blood vessel, while a stenosis is a coarctation of an arterial lumen. Although these vascular abnormalities are very different (opposite) in terms of their morphology, we can identify them using a simple set operation on the two binary image volumes: the pre-treatment vascular volume and the augmented vessel volume.

These two binary volumes are referred to as sets $\mathbb{V}$ and $A$, respectively, which contain only voxels in unity label. As depicted in the small figure of Fig. 2(b), the approximated aneurysmal volume (partition labeled as 2a) can be selected by the user from the set $(\mathbb{V} \backslash A)$, i.e., the surplus volume (partitions labeled as $2 \mathrm{a}$ and $2 b$ ) in the pre-treatment vascular volume with respect to the augmented vessel volume. Whereas the atherosclerotic plaque volume can be approximated from the set $(A \backslash \mathbb{V})$, i.e., the absent vascular volume (partitions labeled as 5).

\section{IMPLEMENTATION ISSUES}

The proposed methodology is implemented using three crossplatform open source C++ software libraries, the Insight Segmentation and Registration Toolkit (ITK) [33], the Visualization ToolKit (VTK) [34], and the wxWidgets (formerly known as wxWindows) [35], for algorithmic computing, visualization and graphical user interface programming, respectively.

\section{A. Centerline Point Selection}

As discussed in Section II-A-1, two points should be selected from the centerline of the disease-free portion of the vessel of interest. To assist the user in selecting those points, we present $\mathbf{M}$ to the user and request the user to select two points on the surface. Then two centerline points can be located by searching the local maxima in the ED map around the two chosen points [36]. We employ the ED transformation [37] available in the VTK [34] and a gradient-ascent strategy to find those local maxima (i.e., the centerline points).

\section{B. Optimization Procedure for Spline Registration}

In a typical optimization procedure, the Cartesian coordinates of all the movable spline knots (henceforth referred to as set $\mathbb{P}$ ) are enumerated to form a parameter vector $\vec{p} \in \mathbb{R}^{3 \cdot \mid}|\mathbb{P}|$. The optimization algorithm then varies $\vec{p}$ to find an energy minimum in a search domain. Nonetheless, $|\mathbb{P}|$ is not fixed in our case, thereby the dimensionality of $\vec{p}$ is one of the parameters to be optimized. To solve this atypical optimization problem, we propose a novel strategy to cope with this iteration-varying dimensionality of the parameter vector.

Algorithm 1 Optimization with Iteration-Varying Dimension

$1: k \Leftarrow 0, E_{k} \Leftarrow+\infty$

2: $\mathbf{C}_{\mathbf{k}} \Leftarrow \operatorname{SPLINE}\left(\mathbf{P}_{\mathbf{a}}, \emptyset, \mathbf{P}_{\mathbf{b}}\right)$

3: repeat

4: $k \Leftarrow k+1$

5: $\mathbb{P}_{k} \Leftarrow\left\{\mathbf{C}_{\mathrm{k}-\mathbf{1}}(i /(k+1)) \mid i \in \mathbb{N}, 1 \leq i \leq k\right\}$, s.t. $\left|\mathbb{P}_{k}\right|=k$

6: $\overrightarrow{p_{k}} \Leftarrow \operatorname{ENUMCOORD}\left(\mathbb{P}_{k}\right)$, s.t. $\overrightarrow{p_{k}} \in \mathbb{R}^{3 k}$

7: $\left\{E_{k}, \overrightarrow{p_{k}^{*}}\right\} \Leftarrow \operatorname{OPTIMIZE}\left(\overrightarrow{p_{k}}\right)$, s.t. $\overrightarrow{p_{k}^{*}} \in \mathbb{R}^{3 k}$

8: $\mathbb{P}_{k}^{*} \Leftarrow \operatorname{REVENUMCOORD}\left(\overrightarrow{p_{k}^{*}}\right)$, s.t. $\left|\mathbb{P}_{k}^{*}\right|=k$

9: $\mathbf{C}_{\mathbf{k}} \Leftarrow \operatorname{SPLINE}\left(\mathbf{P}_{\mathbf{a}}, \mathbb{P}_{k}^{*}, \mathbf{P}_{\mathbf{b}}\right)$

10: until $E_{k}>E_{k-1}$

11: Return $\mathbf{C}_{\mathbf{k}-\mathbf{1}}$

1) Optimization With Iteration-Varying Dimension: Our strategy is given in Algorithm 1. The variable $k$ is an iteration counter, $E_{k}$ stores the energy obtained at the $k$ th iteration, $\mathbf{C}_{\mathbf{k}}$ is the spline obtained after the $k$ th optimization, $\mathbf{P}_{\mathbf{a}}$ and $\mathbf{P}_{\mathbf{b}}$ are the two points selected by the user and $\operatorname{SPLINE}(\cdot)$ returns a spline generated using the knots in the argument list. $\mathbb{P}_{k}$ is the set of $k$ movable knots before the $k$ th optimization, these knots are sampled evenly from the spline obtained in the previous iteration. $\operatorname{ENUMCOORD}(\cdot)$ returns a vector $\overrightarrow{p_{k}} \in \mathbb{R}^{3 k}$ which is the enumeration of the Cartesian coordinates of the points in the given set. OPTIMIZE $(\cdot)$ performs the optimization based on the parameter vector $\overrightarrow{p_{k}}$ and returns a tuple which consists of the minimum energy value obtained at the $k$ th iteration and the corresponding parameter vector $\overrightarrow{p_{k}^{*}}$. REVENUMCOORD $(\cdot)$ reverses the operation of ENUMCOORD $(\cdot)$. This produces a set of knots $\mathbb{P}_{k}^{*}$ from $\overrightarrow{p_{k}^{*}}$ after the $k$ th optimization.

In summary, the algorithm deforms the spline beginning with a single movable knot and performs the optimization iteratively with increasing the number of knots until the minimum energy stops decreasing.

2) Stochastic Optimizer: In this paper, we use the $(1+$ 1)-Evolution Strategy (ES) for the optimization. It is a special type of the Evolutionary Algorithms, kinds of stochastic optimizers, with both the population size and number of children generated equal one [38]. The $(1+1)$-ES has an automatic step size and provides search direction adaptation. It supports parameters with different scaling and has the ability to step out of nonoptimal minima. Therefore, this strategy can give the spline registration problem with a long capture range. One might suggest to use a deterministic optimizer, for instance, gradient descent method, to register the splines because of its absolute repeatability and computational cheapness. We show later experimentally that a gradient descent method gets easily trapped at local minima and, even worse, if the step size is not 
carefully set, it jumps to a nearby local minimum whose energy value is higher than the initial one. Due to these potential problems, we prefer to use $(1+1)$-ES in this paper. Nonetheless, if absolute repeatability is an important issue, a deterministic optimizer could be applied and if it fails, resort to $(1+1)$-ES.

3) Energy Functional Calculation: The weights $\gamma_{\bullet}^{\mathbf{C}}$ in (4) are assumed to be independent of the spline $\mathbf{C}$, i.e., they are kept constant throughout the registration $\left(\gamma_{\bullet}^{\mathbf{C}}=\gamma\right.$. $)$. The derivatives in (5)-(7) can be calculated analytically since we have a closed form of $\mathbf{C}$ [see (1) and (2)]. The first- and second-order derivatives are expressed as

$$
\frac{\partial \mathbf{C}_{i, i+1}(u)}{\partial u}=\left[\begin{array}{llll}
3(u-i)^{2} & 2(u-i) & 1 & 0
\end{array}\right] \mathcal{A P}
$$

and

$$
\frac{\partial^{2} \mathbf{C}_{i, i+1}(u)}{\partial u^{2}}=\left[\begin{array}{llll}
6(u-i) & 2 & 0 & 0
\end{array}\right] \mathcal{A P}
$$

respectively, where $\mathcal{A}$ and $\mathcal{P}$ are the second $4 \times 4$ matrix and third column vector on the right-hand side of (1).

The function $\mathcal{V}(\mathbf{C})$ in (8) is approximated with a circular neighborhood (CN) system [39] [see Fig. 4(a)]. The CN system is composed of concentric circles with the outermost circle's radius equals the local tube radius. The area between two consecutive circles is known as a layer. To be more precise, the radius of the $i$ th circle equals $(i / L) \times R_{\mathbf{C}(u)}$, where $L$ defines number of layers, $i \in \mathbb{N}, 1 \leq i \leq L, R_{\mathbf{C}(u)}$ denotes the tube radius at $\mathbf{C}(u)$ and the first layer is defined by innermost circle. These concentric circles are aligned on a plane with its normal vector parallel to the tangent of $\mathbf{C}$. We sample each concentric circle with $S$ evenly distributed samples. Since the vessel voxels are labeled as ones while the background voxels are labeled as zeros, we determine the vascular structure occupied area in percentage by computing the weighted average label value (a value $\in[0,1])$ at the sample points within the $\mathrm{CN}$ system as follows:

$$
\mathcal{V}(\mathbf{C})=\sum_{i=1}^{L} w_{i}\left(\frac{\sum_{j=1}^{S} v_{i j}}{S}\right)
$$

Weights $w_{i}$ account for the area difference between samples at different layers. They are calculated from the area ratios of each pair of consecutive layers. The area of the $i$ th layer equals $\left(\pi(i)^{2}-\pi(i-1)^{2}\right)$. Therefore, the area ratio $r_{i}$ of the $i$ th layer to the $(i+1)$ th layer is expressed as follows:

$$
r_{i}= \begin{cases}\frac{\pi(i)^{2}-\pi(i-1)^{2}}{\pi(i+1)^{2}-\pi(i)^{2}}=\frac{2 i-1}{2 i+1}, & \text { for } i=1,2, \ldots, L-1 \\ 1, & \text { for } i=L\end{cases}
$$

The weights $w_{i}$ are then calculated as follows:

$$
w_{i}=\prod_{k=i}^{L} r_{k}
$$

In other words, $w_{i}$ denotes the area ratio of the $i$ th layer to the outer layer (i.e., $L$ th layer whose $w_{L}=1$ ). Label value of the

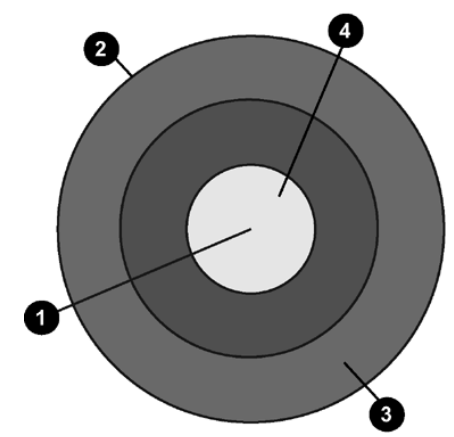

(a)

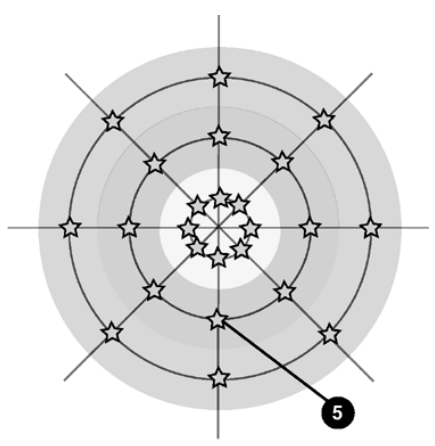

(b)
Fig. 4. Circular neighborhood $(\mathrm{CN})$ system. (a) $\mathrm{CN}$ system is composed of concentric circles (label 1 is the center) with the outermost circle's (labeled as 2 ) radius equals the local tube radius. The area between two consecutive circles is known as layer (layers labeled 3 and 4 are the outermost and innermost layers, respectively). (b) Sample points' locations in a $\mathrm{CN}$ system with $L=3$ and $S=8$. A sample point on the second layer is labeled as 5 .

$j$ th sample point at the $i$ th layer is denoted by $v_{i j}$. The label values are evaluated by the linear interpolation in a continuous space of each layer, or mathematically, on a circle with radius $((i-0.5) / L) \times R_{\mathbf{C}(u)}$ at the sample points of the $i$ th layer. Fig. 4(b) shows sample points' locations in a CN system with $L=3$ and $S=8$ for better illustration. For instance, a unity average label value represents a $100 \%$ vessel occupied area.

Finally, the energy functionals in (5)-(8) are calculated from the derivatives and the function $\mathcal{V}(\mathrm{C})$ with the numerical integration method, two point trapezoidal rule [40]. The series of abscissas used is $\left\{u_{i} \mid u_{i}=i \times \delta u, i \in \mathbb{N}, 0 \leq u_{i} \leq N-1\right\}$, where $N$ denotes the number of (both fixed and movable) spline knots and $\delta u$ is the step size in the parametric space. The step size $\delta u$ should be small enough to produce good approximation of the integration. It is worth noting that the tensile and flexural energy functionals in (5) and (6) can be calculated analytically. This is because the integrands are basically polynomials in degree of 4 and 2, respectively. However, in order to have a consistent way to calculate the overall energy functional for the spline registration, we choose to compute them numerically together with the other analytically nonintegrable functional integrands in (7) and (8).

\section{Initialization of Explicit Vascular Surface Model}

We construct the initial augmented vascular surface $\mathbf{S}_{\mathbf{0}}$ from a binary volume using the Marching Cubes algorithm, with reference to the vessel widths and centerlines determined as described in Section II-A. The binary volume is built by labeling voxels that are laid inside the morphological shape of the augmented vessels with the unity label. It is accomplished by placing solid spheres (filled up with the unity label) with radii equal to one-half of the estimated vessel widths along each centerline. This approach has the advantage of constructing the augmented vessels in a complex vasculature, such as an n-way branching junction. This is because each individual vessel defined by the centerline and the interpolated widths can be easily aggregated together in terms of binary volumes by logical OR operator (treating the unity label as true and the label zero as false). 


\section{Optimization Strategy for Nonrigid Surface Registration}

To minimize the energy functional $E^{\mathrm{S}}$ in (16), a dynamical system governed by the functional is constructed using the Lagrangian mechanics. To achieve this, the functional $E^{\mathrm{S}}$ is reformulated to a notation that is invariant to the surface parameterization. Thus, the derivation of the Euler-Lagrange equations of motion is also independent from the representation of $\mathbf{S}$.

1) The Lagrangian: After the reformulation (see Appendix I), the Lagrangian is written as follows:

$$
E^{\mathbf{S}}=\int_{\mathbf{S}}\left[\sum_{d=1}^{3}\left(\mathcal{E}_{\text {tensile }}^{\mathbf{S}^{(d)}}+\mathcal{E}_{\text {flexual }}^{\mathbf{S}^{(d)}}\right)+\mathcal{E}_{\text {external }}^{\mathbf{S}}\right] d u d v
$$

where

$$
\begin{aligned}
\mathcal{E}_{\text {tensile }}^{\mathbf{S}^{(d)}} & =\lambda_{\mathrm{t}}^{\mathbf{S}}\left|\nabla \mathbf{S}^{(d)}\right|^{2} \\
\mathcal{E}_{\text {flexural }}^{\mathbf{S}^{(d)}} & =\lambda_{\mathrm{f}}^{\mathbf{S}}\left[\left(\nabla^{2} \mathbf{S}^{(d)}\right)^{2}-2 \mathcal{H}\left(\mathbf{S}^{(d)}\right)\right] \\
\mathcal{E}_{\text {external }}^{\mathbf{S}} & =\lambda_{\mathrm{s}}^{\mathbf{S}} \mathcal{E}_{\text {snap }}^{\mathbf{S}}+\lambda_{\mathrm{a}}^{\mathbf{S}} \mathcal{E}_{\text {anti-collapse }}^{\mathbf{S}}
\end{aligned}
$$

The variable $d$ is the dimensionality index, the symbol $\mathbf{S}$ in the integrand is shorthand for $\mathbf{S}(u, v)$ and $\mathbf{S}^{(d)}$ represents the $d$ th component of the vector $\mathbf{S}(u, v)$. The symbols $\nabla, \nabla^{2}$, and $\mathcal{H}\left(\mathbf{S}^{(d)}\right)$ denote the gradient operator, the Laplacian operator, and the determinant of the Hessian matrix of $\mathbf{S}^{(d)}$ on the parametric space, respectively.

2) Euler-Lagrange Equations of Motion: The Euler-Lagrange equations are then obtained using calculus of variations

$$
-\lambda_{\mathrm{t}}^{\mathbf{S}} \nabla^{2} \mathbf{S}+\lambda_{\mathrm{f}}^{\mathbf{S}} \nabla^{4} \mathbf{S}+\nabla \mathcal{E}_{\text {external }}^{\mathbf{S}}=\overrightarrow{0}
$$

where $\nabla^{4}$ denotes the biharmonic operator, square of the Laplacian operator. The matched $\mathbf{S}$ is, therefore, characterized by the equilibrium state of this dynamical system. Through using a gradient-descent method, we can obtain the motion equations for each sample points on $\mathbf{S}$

$$
\begin{aligned}
\mathbf{S}^{(t+\delta t)} & =\mathbf{S}^{(t)}-\frac{\delta t}{\tau} \times \nabla E^{\mathbf{S}} \\
& =\mathbf{S}^{(t)}-\frac{\delta t}{\tau}(\underbrace{\lambda_{\mathrm{t}}^{\mathbf{S}} \nabla^{2} \mathbf{S}^{(d)}}_{\text {tensile force }}-\underbrace{\lambda_{\mathrm{f}}^{\mathbf{S}} \nabla^{4} \mathbf{S}^{(d)}}_{\text {flexural force }}-\underbrace{\nabla \mathcal{E}_{\text {external }}^{\mathbf{S}}}_{\text {external force }})
\end{aligned}
$$

where $\delta t$ is a time step and $\tau$ is a damping coefficient. Assuming the weights in $\mathcal{E}_{\text {external }}^{\mathbf{S}}$ are constants, i.e., $\lambda_{\mathrm{s}}^{\mathbf{S}}=\lambda_{\mathrm{s}}$ and $\lambda_{\mathrm{a}}^{\mathbf{S}}=$ $\lambda_{\mathrm{a}}$, the gradient $\nabla \mathcal{E}_{\text {external }}^{\mathrm{S}}$ is given as follows (see Appendix II for the derivations):

$$
\begin{aligned}
\nabla \mathcal{E}_{\text {external }}^{\mathbf{S}} & =\lambda_{\mathrm{S}} \nabla \mathcal{E}_{\text {snap }}^{\mathbf{S}}+\lambda_{\mathrm{a}} \nabla \mathcal{E}_{\text {anti-collapse }}^{\mathbf{S}} \\
\nabla \mathcal{E}_{\text {snap }}^{\mathbf{S}} & =\left(1-e^{\frac{- \text { ndis }_{\mathrm{M}}(\mathbf{S})}{p}}\right) \times \frac{\mathbf{S}-\mathrm{np}_{\mathrm{M}}^{*}(\mathbf{S})}{\operatorname{ndis}_{\mathrm{M}}^{*}(\mathbf{S})} \\
\nabla \mathcal{E}_{\text {anti-collapse }}^{\mathbf{S}} & =\left(1-e^{-r \times|\Delta \mathbf{S}|}\right) \times \frac{\Delta^{*} \mathbf{S}}{\max (\epsilon,|\Delta \mathbf{S}|)}
\end{aligned}
$$

where $n p_{M}^{*}(\mathbf{S})$ returns $n p_{\mathbf{M}}(\mathbf{S})$ (which gives the nearest point on $\mathbf{M}$ from $\mathbf{S}(u, v)$ ) if ndisM $(\mathbf{S}) \leq R$, otherwise the argument
$\mathbf{S}(u, v)$ is returned. The constant $\epsilon$ denotes a very small positive real number, ndis ${ }_{M}^{*}(\mathbf{S})$ is defined as $\max \left(\epsilon, \operatorname{ndis}_{\mathbf{M}}(\mathbf{S})\right)$ and $\Delta^{*} \mathbf{S}$ equals $\Delta \mathbf{S}$ if $\overrightarrow{n_{0}} \cdot \Delta \mathbf{S} \leq 0$, otherwise it equals $\overrightarrow{0}$.

3) Discrete Representations: In this paper, we assume the weights $\lambda_{\mathrm{t}}^{\mathbf{S}}=\lambda_{\mathrm{t}}$ and $\lambda_{\mathrm{f}}^{\mathbf{S}}=\lambda_{\mathrm{f}}$. The Laplacian and biharmonic terms in (27) are approximated by the umbrella operator [30] at each sample point on $\mathbf{S}$, i.e., each vertex on the triangle mesh, as follows:

$$
\begin{aligned}
& \lambda_{\mathrm{t}} \nabla^{2} \mathbf{S}_{i}=\lambda_{\mathrm{t}} \vec{\alpha}_{i}=\lambda_{\mathrm{t}} \sum_{j \in \mathcal{N}_{i}}\left(\mathbf{S}_{j}-\mathbf{S}_{i}\right) \\
& \lambda_{\mathrm{f}} \nabla^{4} \mathbf{S}_{i}=\lambda_{\mathrm{f}} \sum_{j \in \mathcal{N}_{i}}\left(\vec{\alpha}_{j}-\vec{\alpha}_{i}\right)
\end{aligned}
$$

where $\mathbf{S}_{i}$ represents the $i$ th vertex of the mesh and $\mathcal{N}_{i}$ is the set of neighbors of the vertex $\mathbf{S}_{i}$. We define the function ndism $\left(\mathbf{S}_{i}\right)$ as $\left|\mathbf{S}_{i}-\operatorname{np}_{\mathbf{M}}\left(\mathbf{S}_{i}\right)\right|$. The nearest point on $\mathbf{M}$ is located using a point locator implemented upon an octree-based spatial search available in the VTK [34].

4) Freezing Mechanism: Theoretically, $\mathbf{S}$ should be kept deforming until it converges in a global sense. Nonetheless, it is 1) inefficient to keep moving every mesh vertex at each iteration even when some of them have reached their equilibrium locations and 2) impractical to define a global convergent state since the mesh vertices may jitter around their local minima. Therefore, we propose a freezing mechanism such that the free-form deformation can be speeded up and a convergent state can be defined.

\section{Algorithm 2 Optimization with the Freezing Mechanism}

$1: t \Leftarrow 0, \mathbb{S} \Leftarrow\left\{i \mid \forall i, \mathbf{S}_{i}\right.$ is a vertex of $\left.\mathbf{S}\right\}$

2: $\vec{q}_{i} \Leftarrow \overrightarrow{0}, c_{i} \Leftarrow F, \forall i, \mathbf{S}_{i}$ is a vertex of $\mathbf{S}$

\section{3: repeat}

4: $\mathbf{S}^{(t+\delta t)} \Leftarrow \operatorname{DEFORM}\left(\mathbf{S}^{(t)}, \mathbb{S}\right)$

5: for all $j$ in the set $\mathbb{S}$

6: $\quad \overrightarrow{q_{j}} \Leftarrow\left(\mathbf{S}_{j}^{(t+\delta t)}-\mathbf{S}_{j}^{(t)}\right)+\overrightarrow{q_{j}}$

7: $\quad$ if $\left|\overrightarrow{q_{j}}\right|<Q$ then

8: $\quad c_{j} \Leftarrow c_{j}-1$

9: $\quad$ if $c_{j}<1$ then

10: $\quad \mathbb{S} \Leftarrow \mathbb{S} \backslash\{j\}$

11: end if

12: else

13: $\quad \overrightarrow{q_{j}} \Leftarrow \overrightarrow{0}$

14: end if

15: end for

16: $t \Leftarrow t+\delta t$

17: until $\mathbb{S}=\emptyset$

The mechanism is outlined in Algorithm 2. $t$ is a time variable, $\mathbb{S}$ denotes a set of indices of active vertices, the vector $\overrightarrow{q_{i}}$ 
TABLE I

Parameter Settings of the Proposed Method Used in All Conducted ExPeriments

\begin{tabular}{|c|c|c|c|}
\hline \multicolumn{4}{|c|}{ Centerline Point Selection (cf. Section III-A) } \\
\hline Voxel diagonal length & 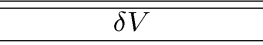 & "Gradient-ascent step size & 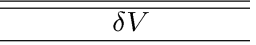 \\
\hline \multicolumn{4}{|c|}{$\begin{array}{l}\text { Spline Registration: (1+1)-Evolution Strategy (cf. Section III-B.2) } \\
\end{array}$} \\
\hline Spline length & 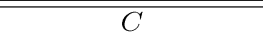 & Shrink factor & $1.1^{-2}$ \\
\hline Growth factor & 1.1 & Parameter scale & 1 \\
\hline Initial radius, $r_{\text {init }}$ & $\delta V$ & Epsilon & $r_{\text {init }} / 2 \times\left|\mathbb{P}_{k}\right|$ \\
\hline Maximum number of iteration & 100 & & \\
\hline \multicolumn{4}{|c|}{ Spline Registration: Discrete Representations (cf. Section III-B.3) } \\
\hline Tensile weight, $\gamma_{\mathrm{t}}$ & 5 & Flexual weight, $\gamma_{\mathrm{f}}$ & 10 \\
\hline Length penalty weight, $\gamma_{1}$ & 1 & Anatomy penalty weight, $\gamma_{\mathrm{a}}$ & 5 \\
\hline Step in the parametric space, $\delta u$ & $\left(\left|\mathbb{P}_{k}\right|-1\right) /\lceil C / \delta V\rceil$ & Number of CN layers, $L$ & 3 \\
\hline Number of samples per layer, $S$ & 16 & & \\
\hline \multicolumn{4}{|c|}{ Surface Registration: Discrete Representations (cf. Section III-D.3) } \\
\hline Time step, $\delta t$ & 1 & $\begin{array}{l}\text { Damping coefficient, } \tau \\
\end{array}$ & 25 \\
\hline Circular snap range, $R$ & $2 \times \delta V$ & Snap energy drop rate, $p$ & $R /(-2 \times \ln (0.01))$ \\
\hline Anti-collapse rate, $r$ & $-2 \times \ln (0.01) / \delta V$ & Tensile force weight, $\lambda_{t}$ & 0.1 \\
\hline Flexural force weight, $\lambda_{\mathrm{f}}$ & 0.2 & Snap force weight, $\lambda_{\mathrm{s}}$ & 1 \\
\hline Anti-collapse force weight, $\lambda_{\mathrm{a}}$ & 5 & & \\
\hline \multicolumn{4}{|c|}{$\begin{array}{l}\text { Surface Registration: Freezing Mechanism (cf. Section III-D.4) } \\
\end{array}$} \\
\hline $\begin{array}{l}\text { Countdown initial value, } F \\
\end{array}$ & 10 & Net displacement threshold, $Q$ & 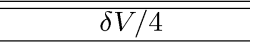 \\
\hline \multicolumn{4}{|c|}{ Surface Registration: Mesh Decimation for Speed Up (cf. Section III-D.4) } \\
\hline Target reduction & $50 \%$ & Triangle aspect ratio & 3 \\
\hline \multicolumn{4}{|c|}{ Transformation of Surface Model into Binary Volume (cf. Section III-E) } \\
\hline Target reduction & $70 \%$ & Topology Preservation & On \\
\hline
\end{tabular}

represents the net displacement vector of the $i$ th vertex in $\mathbb{S}, c_{i}$ is a freezing countdown variable, $F$ is the initial value for the countdown variables and $\mathbf{S}^{(t)}$ denotes the deformed surface at time $t$. The function DEFORM $\left(\mathbf{S}^{(t)}, \mathbb{S}\right)$ moves the vertices of $\mathbf{S}^{(t)}$ whose indices exist in $\mathbb{S}$ according to the motion equations defined in (27). The constant $Q$ defines a threshold for the net displacement. This threshold together with the constant $F$ defines the rate of freezing. The optimization procedure terminates if the set of active vertices is empty. In case it does not happen, the user can preempt it. A more elegant solution to guarantee termination, along the same research line as in [30], is to progressively lower the weights $\lambda_{\mathrm{s}}$ and $\lambda_{\mathrm{a}}$ of the external force.

Further speeding up of Algorithm 2 is possible if one reduces the number of vertices in $\mathbf{S}$, because the worst case time complexity of the algorithm is $O\left(\mathrm{~m}^{2}\right)$, where $m$ is the total number of vertices in $\mathbf{S}$. We employ the implementation of the decimation algorithm [41] available in the VTK [34] to simplify the mesh before the deformation.

\section{E. Transformation of Surface Model Into Binary Volume}

The augmented vascular surface model is represented as a closed triangle mesh. The goal of the transformation is to generate a binary volume for the augmented vessels such that the abnormalities can be identified with a simple set operation (cf. Section II-C). We employ the VTK to convert the triangle mesh into a stencil volume [34]. The stencil is then used to create the desired binary volume with the voxels enclosed by the mesh labeled as ones and the outside voxels labeled as zeros.

It should be noted that if there exists singularities (e.g., two points coincide and a triangle is collapsed into a line) or self-intersecting patches on the surface mesh (usually developed at the boundary of an aneurysmal sac opening), the aforementioned procedure is very prone to unity label leakage. This is because the stencil is built with reference to the surface normals and the surface normals calculated at those problematic regions may not be locally consistent.

To alleviate the possible leakage, the decimation technique based on a quadric error metric [42] is employed to simplify the mesh prior to the transformation. Nonetheless, the new vertices introduced in the decimation algorithm may not be in close proximity to $\mathbf{M}$ [42]. Therefore, we modify the algorithm so as to force the newly introduced vertices to snap to their corresponding nearest points on $\mathbf{M}$ that lay within the range half the voxel diagonal length.

\section{ILlustrations Of THE MethoD}

\section{A. Synthetic Data}

We have tested the proposed method on three synthetic data sets. The parameter settings are listed in Table I, which are found empirically. The design of the synthetic data takes several typical pathologies into consideration: a cerebral aneurysm at the bifurcation of a communicating artery, an abdominal aortic aneurysm (AAA) and a stenosis at the middle cerebral artery (MCA). The synthetic data sets are created as follows. First, circular cross-sectional tubes are employed to model diseasefree lumens geometrically. Then, an ellipsoid is introduced to simulate an aneurysmal sac. In the cases of AAA and arterial stenosis, the tube cross sections are modified to mimic an abnormal localized dilation along an abdominal aorta and a coarctation of an artery, respectively. As a final step, the geometric models are voxelized into binary volumes. The data sets are created to emulate the field of view of the region of interest (6-150 $\mathrm{mm})$ and the image volume $(80 \times 80 \times 120$ vox. $)$ of typical 3 -D angiographies at those anatomical sites. The voxelized synthetic normal lumens (i.e., without introduction of the pathological structures) are treated as the ground truths for evaluation of the proposed method. 


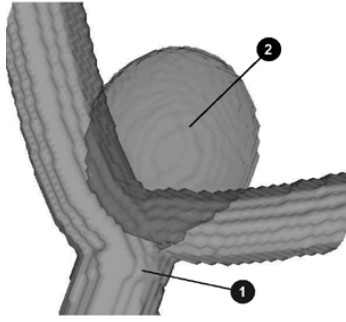

(a)

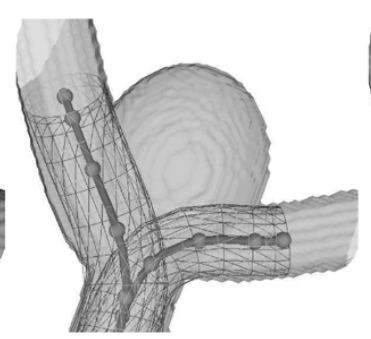

(b)

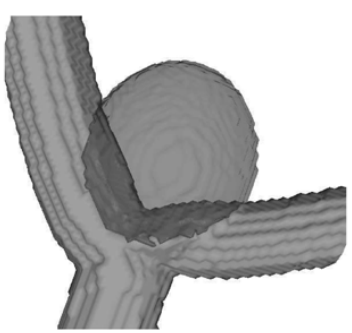

(c)

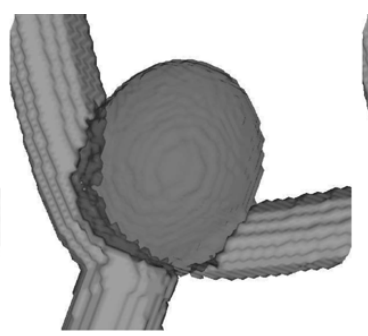

(d)

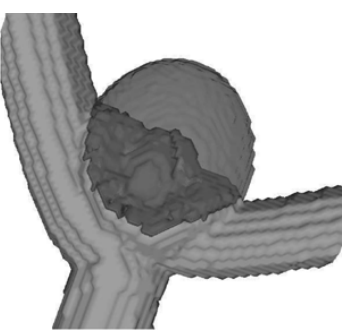

(e)

Fig. 5. Synthetic cerebral aneurysm at the bifurcation of a communicating artery. (a) The ground truth. The solid structures labeled 1 are the synthetic normal lumens. The semi-transparent counterparts labeled 2 are the abnormal volumes corresponding to the aneurysmal lumen. (b) The estimated centerlines of the augmented vessels. They show a high degree of agreement with the synthetic normal lumens [label 1 in (a)]. (c) Posttreatment vessel lumens approximated (solid) and volume of the aneurysmal lumen (semi-transparent) obtained with our method under the condition of a perfect embolization. (d) Results of the methods proposed by (d) Wilson et al. [9], and (e) McLaughlin and Noble [10].

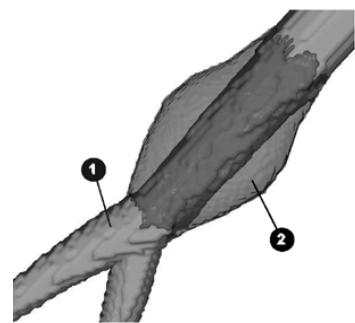

(a)

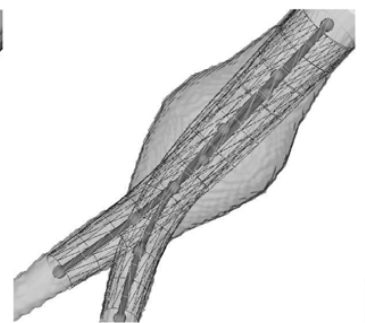

(b)

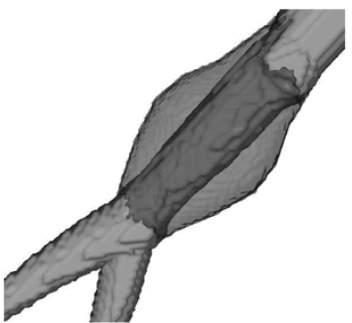

(c)

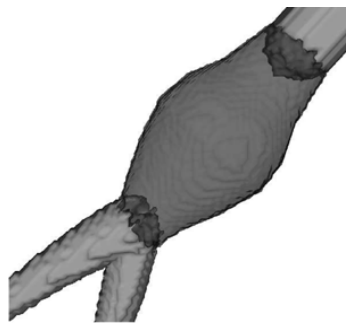

(d)

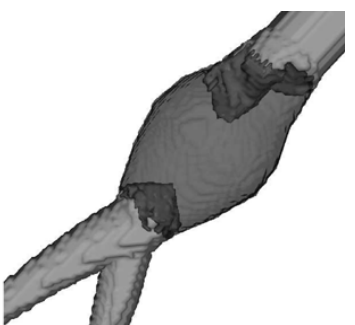

(e)

Fig. 6. Synthetic AAA. (a) The ground truth. The solid structures labeled 1 are the synthetic normal lumens. The semi-transparent counterparts labeled 2 are the abnormal volumes corresponding to the aneurysmal lumen. (b) The centerlines of the augmented vessels estimated. They show a high degree of agreement with the synthetic normal lumens [label 1 in (a)] and can be used to approximate the trajectory of a stent graft in an endovascular treatment. (c) Posttreatment vessel lumens approximated (solid) and volume of the aneurysmal lumen (semi-transparent) obtained with our method under the condition of a perfect occlusion. Results of the methods proposed by (d) Wilson et al. [9] and (e) McLaughlin and Noble [10].

Figs. 5(a), 6(a), and 7(a) and (b) present the synthetic vessel lumens with the ground truths. The solid structures labeled 1 are the normal lumens. The semi-transparent counterparts labeled 2 are the abnormal volumes corresponding to the aneurysmal lumens and the atherosclerotic plaque (that causes a coarctation) of the synthetic lesions. The centerlines of the augmented vessels estimated are depicted in Figs. 5(b), 6(b), and 7(c). They show a high degree of agreement with the synthetic normal lumens. It is noted that the centerlines as shown in Fig. 6(b) canbe used to approximate the trajectory of a stent graft in an endovascular treatment. Moreover, the centerline presented in Fig. 7(c) is not within the confines of the coarctation (highlighted by the arrow). This effectively helps identify the stenosis asymmetry which is a useful clinical parameter in a diagnosis [24]. Volumes of the pathological structures obtained with our method are shown in Figs. 5(c), 6(c), and 7(d) semi-transparently together with the estimates of the vessel lumens after a perfect embolization/occlusion (solid structures in Figs. 5(c) and 6(c) only). Visual comparison suggests that the augmented vessels are capable of modeling the normal lumens and can help identify the pathological volumes.

We have also applied the algorithms proposed by Wilson et al. [9] and McLaughlin and Noble [10] to the two synthetic aneurysms. ${ }^{4}$ The results are shown in Figs. 5(d) and (e) and 6(d) and (e). It is observed that the saccular aneurysmal lumens

${ }^{4}$ We have employed the image inpainting technique proposed in [43] to fill the high-intensity regions in the distance map of the aneurysmal sac center, and use the open mesh of the aneurysmal lumen (the portion of the mesh that does not contact with the augmented vessels) obtained from our method to determine the aneurysmal voxels as suggested in [10] instead of the seed and cull algorithm (since we do not have the implementation).

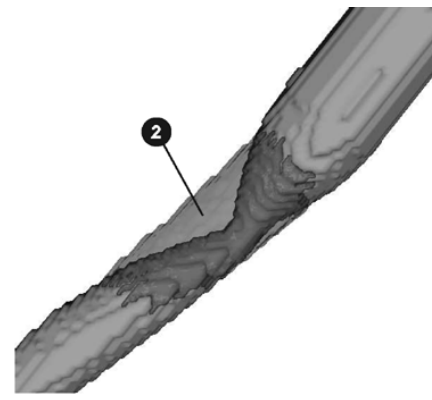

(a)

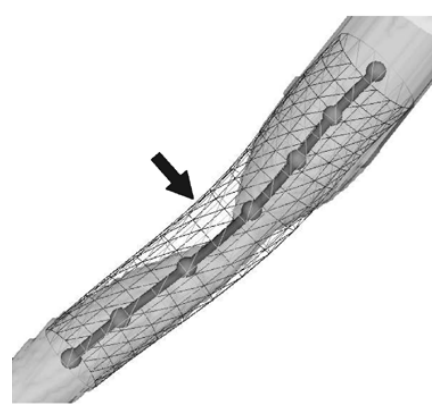

(c)

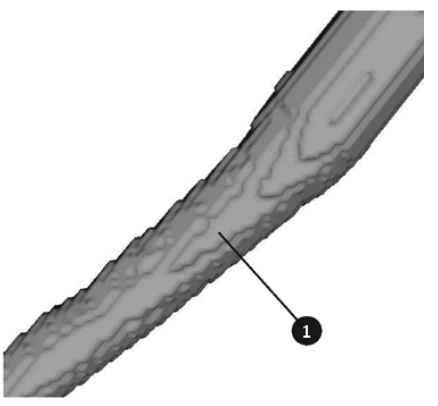

(b)

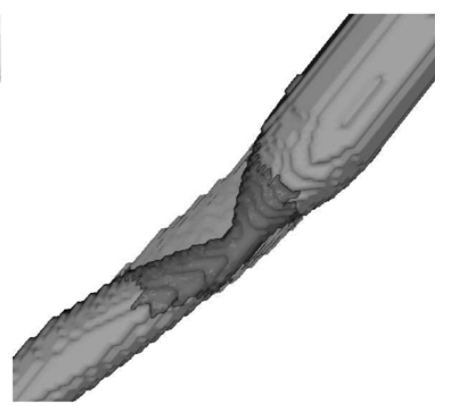

(d)
Fig 7. Synthetic stenosis at the MCA. (a) Synthetic coarctation of MCA (solid structure) and volume of the atherosclerotic plaque of the synthetic lesions labeled 2 (the semi-transparent structure). (b) The ground truth. The solid structure labeled 1 is the synthetic normal lumen. (c) The centerline of the augmented vessel estimated. It shows a high degree of agreement with the synthetic normal lumen [label 1 in (b)] and is not within the confines of the coarctation (highlighted by the arrow). This effectively helps identify the stenosis asymmetry which is a useful clinical parameter in a diagnosis [24]. (d) Volume of the atherosclerotic plaque (semi-transparent) obtained with our method under the condition of a perfect recanalization. 


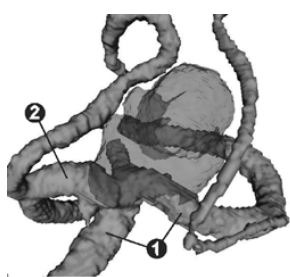

(a)

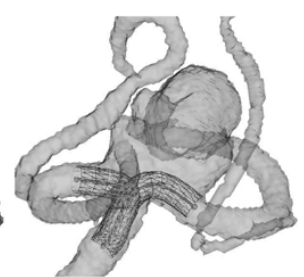

(b)

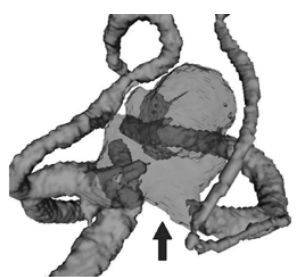

(c)

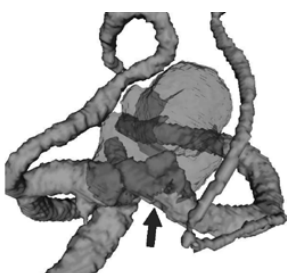

(d)

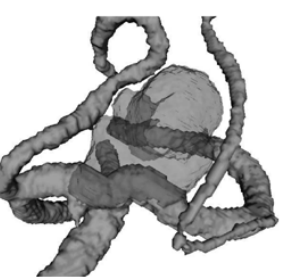

(e)

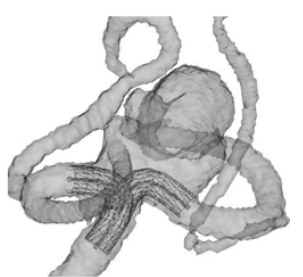

(f)

Fig. 8. (a) Our result on the first clinical data set that contains a wide-neck aneurysm at the bifurcation of ACA labeled 1 and ACoA labeled 2. (b) The centerlines of the augmented vessels estimated. Demarcation of the aneurysmal sac with the methods proposed by (c) Wilson et al. [9] and (d) McLaughlin and Noble [10]. (e) Manually delineated approximation of post-treatment lumens under the condition of a perfect embolization. High similarity between the augmented vessel centerlines and the trajectories of the approximated post-treatment lumens is noticeable. (f) Manually drawn cardinal splines. They are created by inserting additional knots between the two selected end-points for each augmented vessel. The semi-opaque structures in the middle of the aneurysmal sac adjacent to the U-turned portion of ACA are the results of rendering semi-transparent surfaces that occlude other semi-transparent surfaces in the current viewing angle (those semi-transparent surfaces are parts of the aneurysmal sac surface).

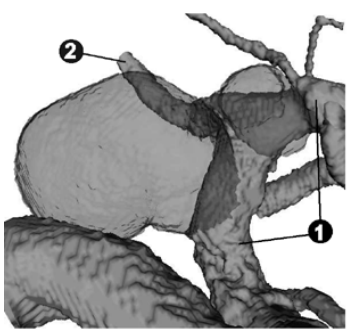

(a)

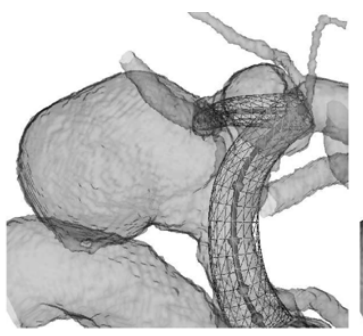

(b)

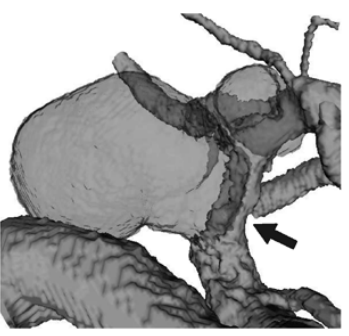

(c)

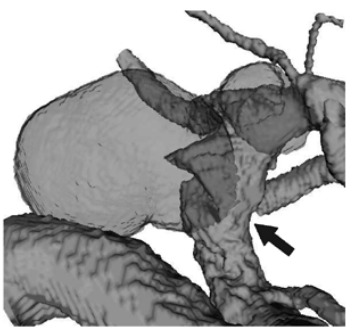

(d)

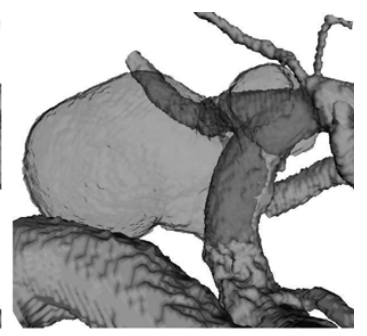

(e)

Fig. 9. (a) Our result on the second clinical data set that contains a more complex lesion (consists of two cerebral aneurysms) at the bifurcation of the ICA labeled 1 and the PCoA labeled 2. (b) The centerlines of the augmented vessels estimated. Demarcation of the aneurysmal sacs with the methods proposed by (c) Wilson et al. [9] and (d) McLaughlin and Noble [10]. (e) Manually delineated approximation of post-treatment lumens under the condition of perfect embolizations. High similarity between the augmented vessel centerlines and the trajectories of the approximated post-treatment lumens is noticeable.

TABLE II

Volumetric ERrors in Percentage With Respect to THE TRUth Pathological (SyNTHETIC Data SETS) OR THE MANUAlly Delineated ABNORMALities (CLINICAL DATA SETS) VOLUMES

\begin{tabular}{lccc}
\cline { 2 - 4 } & $\begin{array}{c}\text { Our } \\
\text { Method }\end{array}$ & $\begin{array}{c}\text { Wilson } \\
\text { et al. [9] }\end{array}$ & $\begin{array}{c}\text { McLaughlin } \\
\text { and Noble [10] }\end{array}$ \\
\hline \hline Synthetic Cerebral Aneurysm & $4 \%$ & $35 \%$ & $28 \%$ \\
Synthetic AAA & $10 \%$ & $50 \%$ & $40 \%$ \\
Synthetic Stenosis & $23 \%$ & - & - \\
\hline $\begin{array}{l}\text { Clinical Data Set 1 } \\
\text { (Cerebral Aneurysm) }\end{array}$ & $2 \%$ & $12 \%$ & $3 \%$ \\
$\begin{array}{l}\text { Clinical Data Set 2 } \\
\text { (Cerebral Aneurysms) }\end{array}$ & $2 \%$ & $6 \%$ & $5 \%$ \\
$\begin{array}{l}\text { Clinical Data Set 3 } \\
\text { (Cerebral Stenosis) }\end{array}$ & $39 \%$ & - & - \\
\hline
\end{tabular}

are likely to be either overestimated or underestimated as compared with the ground truth given in Fig. 5(a). In the case of the fusiform aneurysm, the two methods, by their nature, have difficulties in determining the morphology of the aneurysmal lumen (vascular structure with a cylindrical cavity along its center axis). Volumetric errors in percentage with respect to the truth pathological volumes are listed in Table II. It is evident that the volumetric errors of the results obtained from our method are lower than those from the others.

\section{B. Clinical Data}

Results of our method on three clinical data sets are presented in Figs. 8(a), 9(a), and 10(a) (empirically found parameter settings are listed in Table I). The lesions found in the data sets include 3 cerebral aneurysms and an MCA stenosis. Fig. 8(a) shows a wide-neck aneurysm at the bifurcation of the anterior cerebral artery (ACA) and the anterior communicating artery (ACoA). Figs. 9(a) and 10(a) present a more complex lesion of the junction internal carotid artery (ICA)-posterior commu-

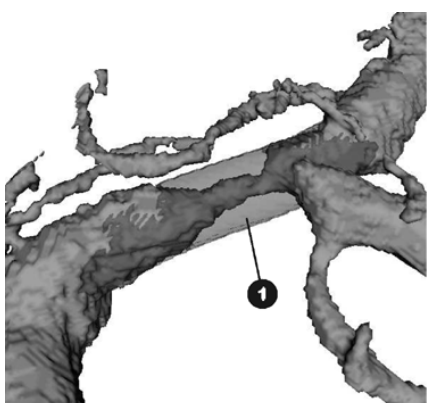

(a)

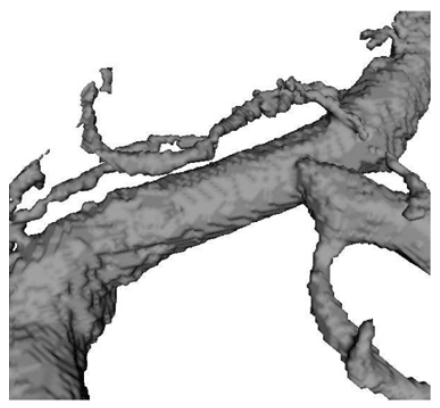

(c)

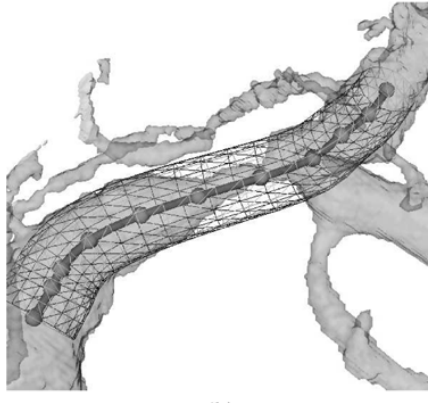

(b)

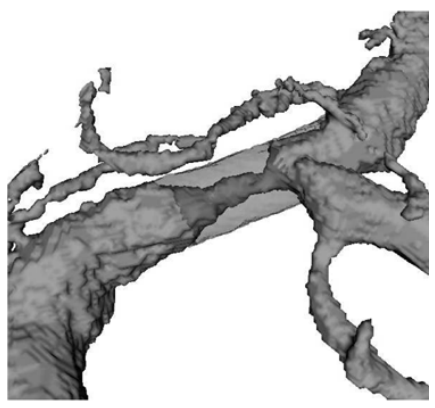

(d)
Fig. 10. (a) Our result on the third clinical data set that contains a coarctation of the MCA, the estimated atherosclerotic plaque volumes are labeled as 1. (b) The centerlines of the augmented vessels estimated. (c) Manual delineation of a perfectly recanalized MCA lumen. (d) Volume of the atherosclerotic plaque deduced from the manually delineated recanalized MCA. It can be noticed that the estimated augmented vessel centerline demonstrated a similar trajectory of the recanalized lumen as the one delineated on a voxel by voxel basis.

nicating artery (PCoA) and a coarctation of the MCA, respectively. These data are 3-D rotational angiographies (RA) acquired by the Philips Integris imager at the Department of Di- 
TABLE III

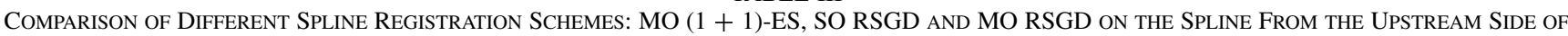
ACA TO THE DOWNSTREAM SIDE

\begin{tabular}{lccccc} 
& \multicolumn{5}{c}{ From the Upstream Side of ACA to the Downstream Side } \\
\cline { 2 - 6 } & $\begin{array}{c}\text { Number of } \\
\text { Movable Knots Used }\end{array}$ & $\begin{array}{c}\text { Value of } \\
\text { Energy Functional }\end{array}$ & $\begin{array}{c}\text { Time Elapsed } \\
\text { (sec.) }\end{array}$ & $\begin{array}{c}\text { Mean of } \\
\text { Errors }\end{array}$ & $\begin{array}{c}\text { Standard Dev. of } \\
\text { Errors }\end{array}$ \\
\hline \hline Manual & 3 & 163 & - & $0 \%$ & $0 \%$ \\
\hline \hline MO (1+1)-ES Run 1 & 4 & 153 & 9 & $28 \%$ & $16 \%$ \\
MO (1+1)-ES Run 2 & 3 & 154 & 4 & $32 \%$ & $21 \%$ \\
MO (1+1)-ES Run 3 & 4 & 155 & 8 & $40 \%$ & $31 \%$ \\
MO (1+1)-ES Run 4 & 4 & 154 & 9 & $38 \%$ & $26 \%$ \\
MO (1+1)-ES Run 5 & 4 & 152 & 7 & $40 \%$ & $26 \%$ \\
MO (1+1)-ES Run 6 & 3 & 154 & 4 & $35 \%$ & $27 \%$ \\
MO (1+1)-ES Run 7 & 4 & 157 & 7 & $24 \%$ & $13 \%$ \\
MO (1+1)-ES Run 8 & 3 & 151 & 5 & $37 \%$ & $21 \%$ \\
MO (1+1)-ES Run 9 & 4 & 155 & 8 & $26 \%$ & $18 \%$ \\
MO (1+1)-ES Run 10 & 4 & 153 & 10 & $33 \%$ & $28 \%$ \\
\hline MO (1+1)-ES Average & - & 154 & 7 & $33 \%$ & $23 \%$ \\
\hline SO RSGD 1 Knot & 1 & 216 & 2 & $67 \%$ & $33 \%$ \\
SO RSGD 2 Knots & 2 & 179 & 3 & $43 \%$ & $41 \%$ \\
SO RSGD 3 Knots & 3 & 229 & 8 & $55 \%$ & $43 \%$ \\
SO RSGD 4 Knots & 4 & 376 & 50 & $516 \%$ & $308 \%$ \\
SO RSGD 5 Knots & 5 & 295 & 19 & $102 \%$ & $94 \%$ \\
\hline MO RSGD & 3 & 158 & 61 & $48 \%$ & $35 \%$ \\
\hline
\end{tabular}

agnostic Radiology and Organ Imaging, Prince of Wales Hospital, Hong Kong. The size of the image volume is $256 \times 256 \times$ 256 vox. with the field of view $30-50 \mathrm{~mm}$.

As depicted in Figs. 8 and 9, similar results are observed as for the synthetic cerebral aneurysm. Our method is capable of producing the aneurysmal volume and the estimated post-treatment lumens [see Figs. 8(a) and 9(a)] that look very similar to the manually delineated (on a voxel by voxel basis) counterparts as shown in Figs. 8(e) and 9(e). The centerlines of the augmented vessels estimated are given in Figs.8(b) and 9(b) for reference. High similarity between these centerlines and the trajectories of the approximated post-treatment lumens that obtained manually is noticeable. On the contrary, Wilson et al. method [9] significantly overestimates the sac volume as a consequence of narrowing/breaking the ACA and McLaughlin and Noble's algorithm [10] slightly underestimates the aneurysmal volume near the sac opening, as highlighted by the arrows in Figs. 8(c) and (d) and 9(c) and (d). For the arterial coarctation data set, our estimated atherosclerotic plaque volume [label 1 in Fig. 10(a)] is comparable to that obtained manually assuming a perfect recanalization [see Fig. 10(d)]. Again the estimated augmented vessel centerline demonstrates a similar trajectory of the recanalized lumen as the one delineated on a voxel by voxel basis shown in Fig. 10(c). Volumetric errors in percentage with reference to the volume of the manually delineated abnormalities are listed in Table II for quantitative comparison. Manual delineation of the pathological structures were performed by an experienced user under the supervision of a consultant radiologist. It is worth mentioning that the manual delineations are not regarded as the ground truths for validation, because they may be produced subjectively.

We have also evaluated our spline registration procedure (i.e., MO approach with $(1+1)$-ES optimization method) for the estimation of augmented vessel centerlines on the first clinical data set (the one shown in Fig. 8). The evaluation is conducted based on the ED error to a manually drawn spline. Two splines are needed for the data set. One fits from the upstream side of
ACA to the downstream side and the other is to ACoA. The two manually drawn splines are shown in Fig. 8(f). They were drawn by inserting additional knots between the two selected end-points for each augmented vessel.

As a comparison, we have tested two other different registration schemes. They are single-order (i.e., fixed number of movable knots) and MO approaches with a regular step gradient descent (RSGD) optimization method. In RSGD method, as the name implies, a regular step size is used in the optimization. The step size is halved if the descending direction is changed more than $90^{\circ}$. This effectively avoids jittering movements around a local optimal. Convergence of RSGD method is denoted by the value of the regular step size. Optimization halts when the step size is below a pre-defined threshold. In the experiments, the threshold was set to one-fourth of the voxel size and the initial value of the step size was five times the size of the voxel size.

Results are listed in Tables III and IV where numbers of movable knots used, values of the energy functional, the time elapsed, means and standard deviations of errors are tabulated. Errors were calculated with respect to the length of the voxel diagonal. It is suggested that single-order (SO) RSGD got easily trapped at local minimum even if the number of movable knots equal to the number of knots inserted manually ( 3 knots). While higher order ( $>3$ movable knots) spline would only worsen the registration, splines with 2 movable knots produced the best results with single-order RSGD. Nevertheless, these results are with higher standard deviations of errors and higher energy values than those from the $\mathrm{MO}$ counterpart. This is because of the low degree of freedom (DOF) of the splines which makes them unable to model portions of the vessel centerlines that exhibit relatively high curvature. MO RSGD seem to be able to alleviate the problem by adaptively increasing the DOF of the cardinal spline. Comparing with the results obtained from MO $(1+1)$-ES, however, those produced by RSGD are less satisfactory. Our optimization scheme is capable of finding less erroneous solutions with respect to the manually drawn splines despite its stochastic nature. It gives spline settings 
TABLE IV

COMPARISON OF DifFERENT SPLINE REGISTRATION SCHEMES: MO $(1+1)$-ES, SO RSGD, AND MO RSGD ON THE SPLINE From the UpSTREAM SidE OF ACA TO ACOA

\begin{tabular}{|c|c|c|c|c|c|}
\hline & \multicolumn{5}{|c|}{ From the Upstream Side of $\mathrm{ACA}$ to $\mathrm{ACoA}$} \\
\hline & $\begin{array}{c}\text { Number of } \\
\text { Movable Knots Used }\end{array}$ & $\begin{array}{c}\text { Value of } \\
\text { Energy Functional }\end{array}$ & $\begin{array}{l}\text { Time Elapsed } \\
\text { (sec.) }\end{array}$ & $\begin{array}{l}\text { Mean of } \\
\text { Errors }\end{array}$ & $\begin{array}{c}\text { Standard Dev. of } \\
\text { Errors }\end{array}$ \\
\hline$\overline{\overline{\text { Manual }}}$ & 3 & 170 & - & $0 \%$ & $0 \%$ \\
\hline MO (1+1)-ES Run 1 & 4 & 172 & 9 & $34 \%$ & $21 \%$ \\
\hline MO (1+1)-ES Run 2 & 4 & 167 & 10 & $37 \%$ & $21 \%$ \\
\hline MO (1+1)-ES Run 3 & 5 & 169 & 13 & $39 \%$ & $19 \%$ \\
\hline MO (1+1)-ES Run 4 & 5 & 171 & 13 & $32 \%$ & $22 \%$ \\
\hline MO (1+1)-ES Run 5 & 4 & 165 & 9 & $28 \%$ & $16 \%$ \\
\hline MO (1+1)-ES Run 6 & 3 & 165 & 5 & $35 \%$ & $20 \%$ \\
\hline MO (1+1)-ES Run 7 & 5 & 169 & 14 & $29 \%$ & $16 \%$ \\
\hline MO (1+1)-ES Run 8 & 4 & 169 & 9 & $37 \%$ & $17 \%$ \\
\hline MO (1+1)-ES Run 9 & 4 & 165 & 10 & $30 \%$ & $18 \%$ \\
\hline MO (1+1)-ES Run 10 & 4 & 172 & 10 & $45 \%$ & $31 \%$ \\
\hline MO (1+1)-ES Average & - & 168 & 10 & $35 \%$ & $20 \%$ \\
\hline SO RSGD 1 Knot & 1 & 191 & 1 & $96 \%$ & $50 \%$ \\
\hline SO RSGD 2 Knots & 2 & 179 & 8 & $67 \%$ & $48 \%$ \\
\hline SO RSGD 3 Knots & 3 & 180 & 19 & $81 \%$ & $48 \%$ \\
\hline SO RSGD 4 Knots & 4 & 198 & 30 & $133 \%$ & $81 \%$ \\
\hline SO RSGD 5 Knots & 5 & 220 & 40 & $255 \%$ & $161 \%$ \\
\hline MO RSGD & 3 & 173 & 8 & $57 \%$ & $36 \%$ \\
\hline
\end{tabular}

with lower energy values, smaller means and smaller standard deviations of errors in all the 20 runs than those given by the RSGD method. Indeed it is the stochastic property that offers $(1+1)$-ES the ability to step out of nonoptimal minima.

Computational-wise, MO $(1+1)$-ES is believed to be more expensive than RSGD owing to its nondeterministic nature. However, in this application, it does not harm the performance as the processing time is down to seconds. $(1+1)$-ES took $<15 \mathrm{~s}$ to register the cardinal splines with variable number of knots on a $2 \mathrm{GHz}$ PC. In contrast, MO RSGD may waste time in jumping to a nearby local minimum which has a higher energy value due to the facts that the energy functional is highly nonconvex with numerous local energy minima and the initial regular step size is relatively too large. This is observed during the registration of the cardinal spline onto ACA. RSGD took $61 \mathrm{~s}$ (8 times that $(1+1)$-ES had spent) to find a fair spline setting. We found that it wasted $54 \mathrm{~s}$ to find a local minimum whose energy value is higher than the initial one when the third movable knot was inserted. On the other hand, $(1+1)$-ES did not have this problem, thanks to the automatic step size and search direction adaptation of the algorithm.

\section{In Vivo Evaluations}

Validation of the proposed method is difficult. Even if we have a physical lumen phantom of a lesion, not to mention patient data, we still cannot verify the correctness of the identified abnormal volumes. This is because the diseases would have already changed the lumen appearance, making it deviated from the disease-free lumen even though it is repaired by an endovascular treatment. Manual delineation of the abnormal volumes seems to be an option. Despite the fact that it is a very challenging task, as mentioned in Section IV-B, such operation may be highly subjective. Therefore, it is not encouraged to define correctness upon the manually delineated volumes. Because of this, we propose a novel in vivo study to evaluate the abnormalities obtained from our method in an indirect fashion, which exploits the fact that the abnormalities are complementary to the augmented vessels. By verifying a high similarity between the augmented vessels and several disease-free lumens at the same anatomical location, the identified abnormal lumens are then believed to be satisfactory. In addition, we also assess the applicability of the proposed method to the measurements of several clinical parameters.

We have applied the method (see Table I for the parameter settings) to 17 3-D RA data sets, including seven aneurysms at the junction ACA-ACoA in six cases, ten ICA-PCoA aneurysms in nine cases and two MCA stenoses. These data sets were all acquired by the same protocol and machinery as described in Section IV-B.

\section{A. Comparisons to Disease-Free Lumens}

Since arterial bifurcations are common sites of cerebral aneurysms [44], we have chosen to compare the augmented vessels to the corresponding disease-free lumens at the ACA-ACoA and ICA-PCoA junctions. We quantify a bifurcation vasculature with a distance function $\mathcal{D}(\phi, \theta)$ of its junction center. The center is determined from the local maximum in the ED map (of the augmented vessels or the disease-free lumens) around the bifurcation. The function $\mathcal{D}$, along the same research line as in [8], is built upon a spherical coordinate system, where $\phi$ and $\theta$ are the polar and the azimuthal angles, respectively. We match the south pole $(\phi=\pi)$ and the half-plane $\theta=0$ of the system to the upstream and the downstream directions of the cerebral arteries, respectively, as depicted in Fig. 11(a).

Two distance functions of disease-free ACA-ACoA bifurcations (hereafter referred to as $\mathcal{D}_{\text {df }}$ ) are shown in Fig. 11(b) and (c). It is believed that they share high similarity, in which three high-intensity regions characterize the upstream (region 1) and two downstream (regions 2 and 3) arterial lumens. Fig. 11(d) and (e) presents the distance maps of a disease bifurcation and the corresponding augmented vessels (henceforth referred to as $\mathcal{D}_{\mathrm{d}}$ and $\mathcal{D}_{\mathrm{a}}$ ). It is observed that there is a noticeable high-intensity region (region 4) toward the north pole area (due to the 


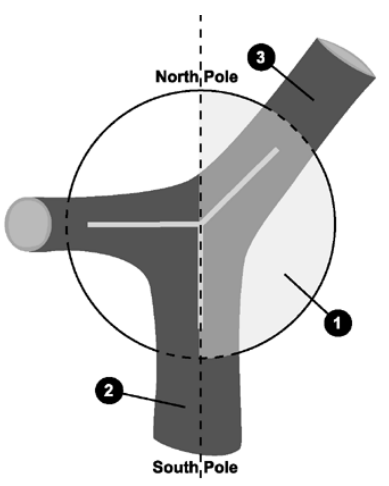

(a)

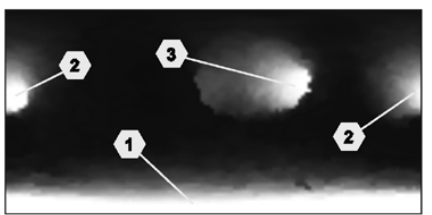

(b)

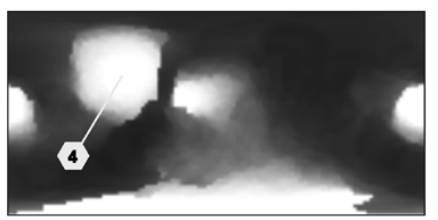

(d)

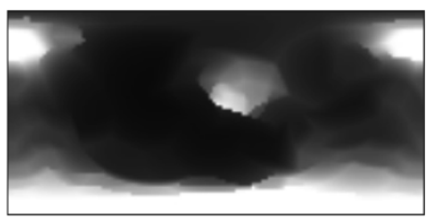

(c)

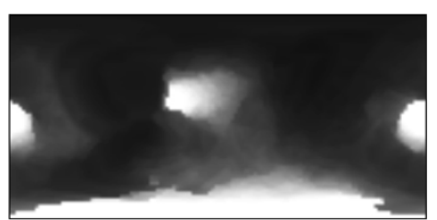

(e)
Fig. 11. Spherical coordinate system and distance functions. (a) The south pole $(\phi=\pi)$ and the half-plane $\theta=0$ labeled 1 of the system are matched to the upstream labeled 2, and the downstream labeled 3 directions of the cerebral arteries, respectively. Distance functions of ACA-ACoA bifurcations of (b)-(c) disease-free lumens, (d) diseased lumens, and (e) augmented vessels. The origin ( $\phi=0, \theta=0$ ) of the distance function is at the upper-left corner of the distance image. Region 1 corresponds to the upstream arterial lumen, regions 2 and 3 denote the downstream lumens, and region 4 is due to the existence of an aneurymsal sac.

existence of an aneurysmal sac) in $\mathcal{D}_{\mathrm{d}}$, and $\mathcal{D}_{\mathrm{a}}$ is very similar to $\mathcal{D}_{\mathrm{df}}$.

Thus, we exploit these properties to justify that our augmented vessels are similar to the disease-free lumens and, therefore, are good estimates of the post-treatment lumens. The similarity is calculated based on the distance function. Given a set of $\mathcal{D}_{\mathrm{df}}$ from a particular anatomical location (hereafter referred as to $\left\{\mathcal{D}_{\mathrm{df}}\right\}$ ), we compute a subspace with principal component analysis (PCA) that can represent $\mathcal{D}_{\mathrm{df}}$ generally with several principal components. The absolute error $\xi$ of $\mathcal{D}$ to that disease-free subspace can then be defined. It is the absolute ED from $\mathcal{D}$ to its projection on the subspace. Such computation is very typical in pattern classification [45]. Intuitively, the similarity can be calculated as a zero-mean Gaussian function of $\xi$. However, due to there may have high variability amongst $\mathcal{D}_{\mathrm{df}}$ and the size of $\left\{\mathcal{D}_{\mathrm{df}}\right\}$ is relatively small, we propose to quantify the similarity with hypothesis tests on the distribution of $\xi$ instead. By applying leave-one-out technique, we obtain a set of $\xi_{\mathrm{df}}$, the absolute errors for $\left\{\mathcal{D}_{\mathrm{df}}\right\}$. Then we compare if the absolute errors calculated from $\mathcal{D}_{\mathrm{d}}$ and the corresponding $\mathcal{D}_{\mathrm{a}}\left(\xi_{\mathrm{d}}\right.$ and $\xi_{\mathrm{a}}$, respectively) are from the same distribution as of $\xi_{\mathrm{df}}$. Distance functions that have $\xi$ with distribution which is
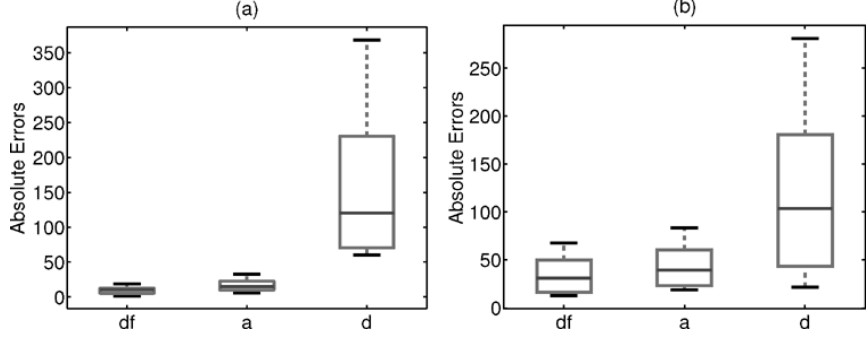

Fig. 12. Absolute errors $\xi_{\mathrm{df}}, \xi_{\mathrm{a}}$ and $\xi_{\mathrm{d}}$ for the junctions (a) ACA-ACoA and (b) ICA-PCoA.

TABLE V

Results of THE Hypothesis Testing (SignificANT LEVEL $\alpha=0.02$ )

\begin{tabular}{lcc} 
& $\begin{array}{c}\text { Junction } \\
\text { ACA-ACoA }\end{array}$ & $\begin{array}{c}\text { Junction } \\
\text { ICA-PCoA }\end{array}$ \\
\hline \hline$k_{\mathrm{df}}$ & 9 & 6 \\
$k$ & 6 & 9 \\
t-value of $H_{0}: \overline{\xi_{\mathrm{df}}}=\bar{\xi}_{\mathrm{a}}$ & 2.0421 & 0.9478 \\
t-value of $H_{0}: \bar{\xi}_{\mathrm{df}}=\bar{\xi}_{\mathrm{d}}$ & 4.5012 & 2.6331 \\
$t\left(\alpha / 2, k_{\mathrm{df}}+k_{\mathrm{df}} \times k-2\right)$ & 2.3890 & 2.3920 \\
\hline \hline \multicolumn{2}{c}{ t-value $<t(\cdot) \Rightarrow$ Do Not Reject $H_{0}: \bar{\xi}_{\mathrm{df}}=\bar{\xi}_{\mathrm{a}}$} \\
\multicolumn{2}{c}{ t-value $>t(\cdot) \Rightarrow$ Reject $H_{0}: \overline{\xi_{\mathrm{df}}}=\overline{\bar{\xi}_{\mathrm{d}}}$} \\
\hline
\end{tabular}

the same as that of $\xi_{\mathrm{df}}$ are suggested to be similar to $\mathcal{D}_{\mathrm{df}}$. The following steps summarize the justification procedure:

Step 1) Suppose we have $k_{\mathrm{df}}$ disease-free bifurcations and $k$ disease bifurcations (or augmented vessels). Compute all the values of $\mathcal{D}_{\mathrm{df}}, \mathcal{D}_{\mathrm{d}}$, and $\mathcal{D}_{\mathrm{a}}$.

Step 2) Truncate $\mathcal{D}$. to ignore the region $(2 / 3) \pi \leq \phi \leq$ $\pi$, limit the maximum values in $\mathcal{D}$. to $5 \mathrm{~mm}$, normalize and smooth $\mathcal{D}$. with a Gaussian kernel $(\sigma=$ $(3 / 32) \pi)$. This helps eliminate the upstream and downstream vessel width variations and the effect of different vessel curvatures in the comparisons.

Step 3) Perform the leave-one-out PCA on $\left\{\mathcal{D}_{\mathrm{df}}\right\}$ to obtain $k_{\mathrm{df}}$ sets of principal components.

Step 4) For each set of the principal components, calculate the absolute errors $\xi$. of the left-out $\mathcal{D}_{\mathrm{df}}, \mathcal{D}_{\mathrm{d}}$, and $\mathcal{D}_{\mathrm{a}}$ (i.e., the absolute $\mathrm{ED}$ from $\mathcal{D}$. to their projections on the $\left(k_{\mathrm{df}}-1\right)$ dimensional subspace). In total, we have $k_{\mathrm{df}}$ absolute errors $\xi_{\mathrm{df}}$ and $\left(k_{\mathrm{df}} \times k\right)$ absolute errors $\xi_{\mathrm{d}}$ and $\xi_{\mathrm{a}}$.

Step 5) Study and check if $\xi_{\mathrm{d}}$ and $\xi_{\mathrm{a}}$ come from the distribution of $\xi_{\mathrm{df}}$.

Fig. 12 presents two box-and-whisker diagrams of the absolute errors $\xi$ for the junctions ACA-ACoA and ICA-PCoA. It appears that $\xi_{\mathrm{df}}$ and $\xi_{\mathrm{a}}$ come from the same population, whereas $\xi_{\mathrm{d}}$ is from a different one. We have performed two t-tests 5 to justify our claim for each junction. The two null hypotheses are 1) $H_{0}: \bar{\xi}_{\mathrm{df}}=\bar{\xi}_{\mathrm{a}}$ and 2) $H_{0}: \bar{\xi}_{\mathrm{df}}=\bar{\xi}_{\mathrm{d}}$. The significance level is $\alpha=0.02$ and two-sided tests are used. The results are listed in Table $\mathrm{V}$. We found that there is insufficient evidence to reject the first $H_{0}$ but not for the second one. Therefore, we believe that our augmented vessels are good estimates of the post-treatment

${ }^{5} \mathrm{~A}$ t-test determines whether the two sets of measured values differ from each other in a significant way under the assumptions that the underlying distributions are normal and the their variances are about equal [46]. 


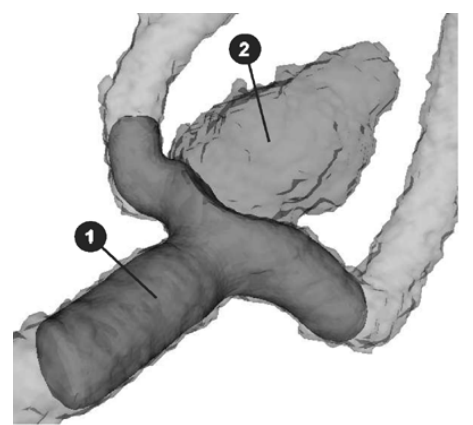

(a)

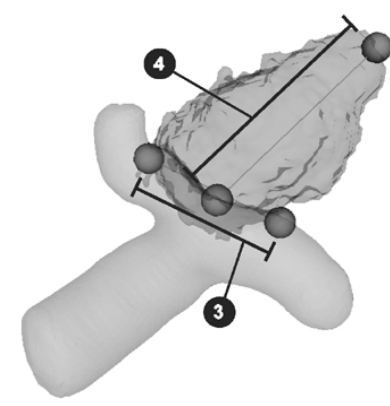

(b)

Fig. 13. Measurements of a demarcated aneurysmal lumen. (a) Augmented vessels labeled 1 help demarcate an aneurysmal lumen labeled 2 from the vascular structures. (b) Measurements of the neck width $N_{\mathrm{D}}$ labeled 3 and the dome height $D_{\mathrm{H}}$ labeled 4 on the detached aneurysmal lumen.

lumens (obtained from perfect embolization/occlusion/recanalization) at $98 \%$ confidence level.

\section{B. Measurements of Clinical Parameters}

Aneurysmal neck diameter $N_{\mathrm{D}}$ (maximum width of the opening of an aneurysm) and dome height $D_{\mathrm{H}}$ (distance between the opening and the fundus, the farthest portion of the sac boundary from its opening) are important clinical parameters of an endovascular embolization [47]. In this study, we show that measurements of those two parameters are more repeatable in terms of intraoperator and interoperator variabilities if the aneurysmal volume is identified. Fig. 13(a) shows a demarcated aneurysmal lumen (label 2) with a well-defined neck surface (the boundary between the lesion lumen and the augmented vessels which are labeled as 1). The parameters $N_{\mathrm{D}}$ and $D_{\mathrm{H}}$ can then be measured with ease if the measurements are taken with reference to this neck surface, as illustrated in Fig. 13(b).

We have asked two experienced operators to perform the measurements on the 17 aneurysmal sacs in the 15 cases with and without the application of our method (demarcation of the aneurysmal sacs). The lumens to be measured were presented to the operators in random orders. One of the operators was requested to perform the measurements twice with two days' period time to disregard any bias toward the second measurements.

Fig. 14 shows four Bland and Altman plots [48] (i.e., of the differences between the two measurements against their means) to assess the agreement between the intraoperator and interoperator measurements of the parameters $N_{\mathrm{D}}$ and $D_{\mathrm{H}}$. The coefficients of repeatability $(2 \times$ standard deviation of the measurement differences) are listed in Table VI. It is evident that 1) the intraoperator and interoperator variabilities are roughly the same regardless of the demarcation of abnormalities and 2) the coefficients of repeatability are reduced by $10+$ times if the measurements are taken with the demarcation of the sacs (see also the decrease in the spread of the data points in the plots). This implies that our method can help increase the repeatability of the measurements significantly.

Another clinical parameter for an endovascular treatment is the stenosis severity. It is commonly measured using the NASCET index that is based on the minimal diameter of

(a)

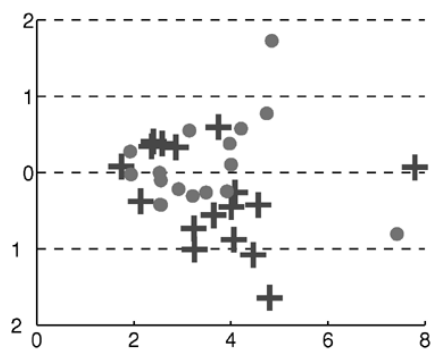

(c)
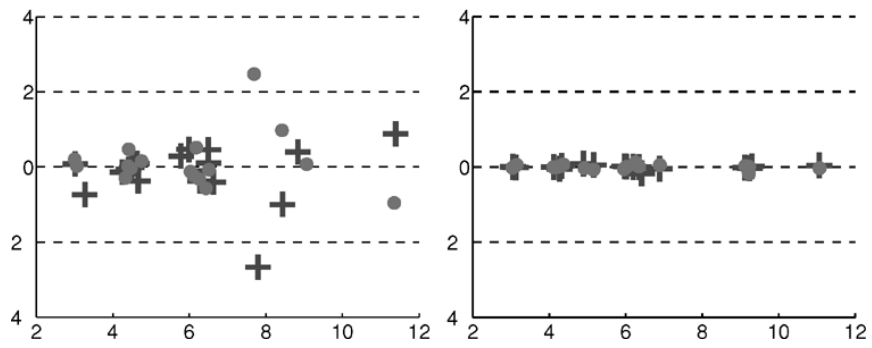

Fig. 14. Bland and Altman plots [48] (axis unit is in mm, mean measurements are in $\mathrm{X}$-axis and measurement differences are in $\mathrm{Y}$-axis) of the measurements of (a) neck widths on the vascular lumens, (b) neck widths on the demarcated aneurysmal lumens, (c) dome heights on the vascular lumens and (d) dome heights on the demarcated aneurysmal lumens in the 153-D RA cases by the two experienced operators. The crosses + and the dots $\bullet$ represent the data points of the intraoperator and the interoperator measurements, respectively.

TABLE VI

COEFFICIENTS OF REPEATABILITY OF $N_{\mathrm{D}}$ AND $D_{\mathrm{H}}$ IN MiLlimeters

\begin{tabular}{llcc}
\cline { 3 - 4 } & & $\begin{array}{c}\text { Without } \\
\text { Demarcation }\end{array}$ & $\begin{array}{c}\text { With } \\
\text { Demarcation }\end{array}$ \\
\hline \hline$N_{\mathrm{D}}$ & Intra-operator & 1.2627 & 0.1227 \\
& Inter-operator & 1.1802 & 0.1041 \\
\hline$D_{\mathrm{H}}$ & Intra-operator & 1.5814 & 0.1088 \\
& Inter-operator & 1.4841 & 0.1279 \\
\hline
\end{tabular}

the stenotic lumen and the mean diameter of the nonstenotic lumen (either proximal or distal to the coarctation) [49]. This measurement, however, assumes circularity in the vascular cross sections. Thus, the measurement based upon irregular cross-sectional areas is developed [7] to suit the clinical situation. Nonetheless, possible bifurcations, prestenotic and poststenotic dilations that are close to the lesion site may further complicate the measurement of severity. With the approximation of the atherosclerotic plaque volumes, our method allows in-site measurements at the severe regions (as depicted in Fig. 15) which can increase the measurement repeatability.

\section{DISCUSSIONS}

\section{A. Representation of Deformable Model}

Surface model is a commonly used deformable model representation found in literature [6], [13]-[18], [23], [50]. As the name implies, the deformable model is represented semantically in terms of surface, though syntactically it may be defined by a variety of primitive tuples, for instance, cores (medial loci) [23], [50], m-reps [6], and medial axis-radius tuples [13]-[18].

Cores are considered as the medial loci of a 2-D/3-D object [50]. They are invented to extract medial loci from gray-scale 


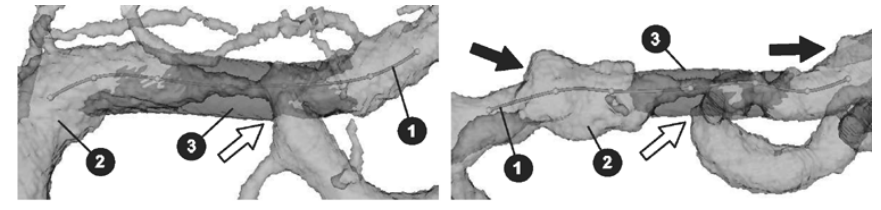

(a)

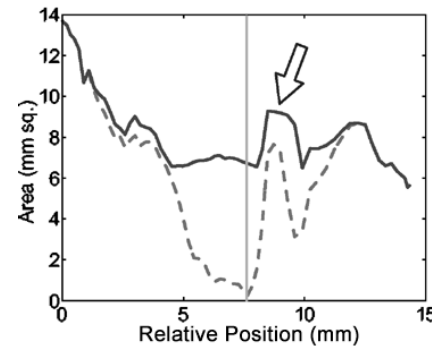

(c) (b)

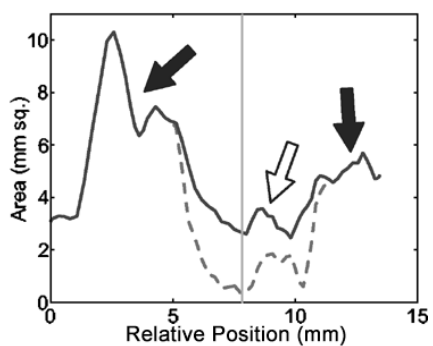

(d)
Fig. 15. Stenotic lumens, the estimated atherosclerotic plaque volumes and the cross-sectional areas along the manually selected vessel centerlines labeled 1. (a)-(b) Two stenotic lumens labeled 2 proximal to the first bifurcation at the MCA in two 3-D RA cases together with the estimated atherosclerotic plaque volumes labeled 3. (c)-(d) The cross-sectional areas of the stenotic lumens (dotted line) and the estimated recanalized lumens (solid line), i.e., the stenotic lumen volume plus the estimated atherosclerotic plaque volume. The hollow arrows highlight the bifurcations. The solid arrows highlight the prestenotic and poststenotic dilations. These are the regions that may further complicate the measurement of stenosis severity. The vertical straight lines in the middle of the plots indicate the in-site measurements at the severe regions.

images, which are invariant to translation, rotation and zoom. As such, cores are good for shape description and analysis in images. They can also help represent object surfaces by defining boundary vertices that are protruded from the medial loci extracted. In a later publication, Pizer et al. [6] presented an extension of the core, namely m-rep, aiming at describing complex shapes in 3-D that are capable of capturing prior geometric information primarily for segmentation with deformable models. The m-rep defines shapes in a more precise fashion. Besides the medial axis position, it uses three extra parameters: a width that denotes the distance between the medial point and its boundary vertices, a local coordinate frame and an "object angle" that defines the local narrowing ratio of the shape [6]. In order to model a 3-D object, a sheet of m-reps is needed.

Nevertheless, the vessels in human bodies are generally tubular in shape. It is more intuitive to model them in terms of the medial axis-radius tuples (i.e., circular cross-sectional tubes) [13]-[18] rather than the medial sheet in the m-rep. The medial axis-radius tuple representation does, however, suffer from surface self intersections if the vessel curvature is high [13]. As such, Yim et al. [14] proposed a modified cylindrical coordinate system to solve this problem, which warps the radial lines based on a merging mechanism to avoid surface self intersections.

The pitfall of building a model upon the medial axis-radius tuples is that it can neither represent complex vasculatures (e.g., vessel branches) nor support the deformation of multiple connected vessels with a single deformable surface model. This is because each vessel has to be represented by a separate set of tuples and, hence, a different surface. As such, we use a triangle mesh for the model representation and embed the shape priors in the initialization and the energy functionals of the nonrigid surface registration problem. This allows us to decouple the shape priors from the model representation and keep the deformable model as simple as possible.

\section{B. Parametric Model of Vessel Centerlines}

Tracking the center axes of body vessels is one of the fundamental procedures in vascular image analysis [17]. It is commonly resolved by tracing the medial axis iteratively given a manually selected seed point from a vascular tree. The medial axis are determined by either evaluating the intensity gradient magnitudes [17], [22], the medialness measure [50], [51], and the vesselness metric [13], [18] (up to a scaling factor which characterizes the local vessel width), or finding the local maxima in the ED map if the vascular segmentation is known.

However, as revealed in [22] and [52], such approach needs special cares at bifurcations, joining vessels, locally wide and asymmetric vessels (e.g., aneurysms) to avoid axis distortion or making a turn in the tracking process. In addition, at a severe stenotic lumen where the vessel is too narrow to be tracked, there may be no trace of the medial axis at all. To get rid of all these problems, we suggest to use a fixed end parametric curve to model the augmented vessel centerline. Having taken into account the volumetric information and the curve regularity, we propose a novel methodology (cf. Sections II-A-3 and III-B) to track the augmented vessel centerlines that are deliberately different from the medial axes of the diseased lumens (for instance, asymmetrical aneurysmal and stenotic lumens) obtained from all the aforementioned algorithms (the differences between the estimated centerlines and the ridges of the ED map are shown in Fig. 1).

Relating the physical and the physiological properties of blood vessels to the vessel centerline model is difficult, if not impossible. Despite a thorough understanding of the blood vessel wall composition [26], to the best of our knowledge, there is no direct study of the relationship between those properties and the intrinsic properties (i.e., stiffness and rigidity) of the vessel centerlines. Noted that the high level attributes of blood vessels, for instance, tortuosity [53], might have been studied. In all the experiments conducted, we experienced that the chosen spline is indeed sufficient for the augmented vessel axis modeling.

The choice of the zero-tension cardinal spline is, however, an implementation preference. We choose the cardinal splines, instead of the conventional open B-splines as used in [9], [13], is that the cardinal splines have the advantage of being able to pass through the knots, which makes the representation more intuitive both in the estimation of the tube centerline as well as the visualization of the tube and the knots.

\section{Estimation of Local Vessel Widths at Centerline End-Points}

Poor approximation of the local vessel widths at the centerline end-points may be obtained if the lumen cross section deviates far from that being circular. This may be the case in venous lumens [26]. Veins have thinner walls which, compared to arteries, may collapse under external tissue pressure. The valves present in the veins change the shape of the lumens as well. Nevertheless, it is rare in the disease-free arterial lumens found in 
the testing data sets. This is because arteries have thicker walls which maintain their shape even when fluid pressure is reduced and they have no internal structure that makes the lumen cross sections deviated significantly from circles [26].

\section{Selection of Parameters of Spline and Surface Registrations}

The parameters of the spline and surface registrations listed in Table I are found empirically. However, six of them are derived from the voxel diagonal length $\delta V$, the spline length $C$ and the number of knots in the spline $\left|\mathbb{P}_{k}\right|$. The voxel diagonal length is the longest distance between neighboring voxels in the 26-neighborhood system. We initiate $(1+1)$-ES with an initial search range (the initial radius, $r_{\text {init }}$ ) that is as large as the 26-neighborhood system for each movable knot. Furthermore, we terminate it if the change in magnitude of the parameter vector $\vec{p}$ is less than $r_{\text {init }} / 2 \times\left|\mathbb{P}_{k}\right|$ (the value of the epsilon of $(1+1)$-ES). This implies the optimization halts when the displacements of the movable knots are less than half of $r_{\text {init }}$ on average. In the numerical integration of the energy functional for the spline registration, the step size in the parametric space $\delta u$ is set such that the spline is sampled along its length with distance approximately $\delta V$ apart, therefore, $\delta u=\left(\left|\mathbb{P}_{k}\right|-1\right) /\lceil C / \delta V\rceil$. Values of the snap energy drop rate $p$ and anti-collapse rate $r$ of the surface registration are also set on purpose. Their values are determined such that the magnitudes of the corresponding forces reach their maximum magnitudes $(=1)$ when $\operatorname{ndis}_{\mathbf{M}}(\mathbf{S})$ and $|\Delta \mathbf{S}|$ are greater than half of the circular snap range and the voxel diagonal length, (i.e., $R / 2$ and $\delta V / 2$ ), respectively. ${ }^{6}$

\section{E. Limitations of the Method}

Although the approximations of the augmented vessel centerlines are dependent on the selections of their end-points (cf. Section II-A-3), in all the experiments conducted, we noted that this approximation is insensitive to the choice of those points as long as this rule of thumb is satisfied: the end-points are selected at the immediate disease-free proximal and distal regions of the lesion site. The only exceptional cases are if 1 ) the end-point is selected at a branching junction and 2) the initial spline defined by the two chosen end-points passes through objects of no interest. In these cases, the estimated augmented vessel centerline may deviate from the desired centerline. It is because of the effect of the branches on the local maxima in the ED map [52] and the $(1+1)$-ES may get trapped at a local minimum in the spline registration. Users should avoid these cases when they select the centerline end-points.

Moreover, the vessel width assumption (i.e., the vessel widths change linearly along the vessel centerline) may not be valid in cases where the lumen size of the vascular structures suddenly decreases. An example of this is the segment originated from the ICA toward the PCoA in the circle of Willis, where the size of the PCoA could be quite variable [54]. One solution may be to allow manual adjustment in the estimated width of the upstream vessel (ICA) to compensate the sudden decrease in the vessel size.

\footnotetext{
${ }^{6}$ The snap force $\nabla \mathcal{E}_{\text {snap }}^{\mathbf{S}}$ in (29) does vanish if $\operatorname{ndis}_{\mathbf{M}}(\mathbf{S})>R$ indeed.
}

Regarding the limitations in terms of pathology, amongst the 17 3-D RA data sets that we have tested, we encounter no difficulties in identifying the volumes of the pathological structures. However, our method may have problem in detaching: 1) an aneurysmal sac which has a neck diameter much greater than its dome height (i.e., $N_{\mathrm{D}} \gg D_{\mathrm{H}}$ ) and the dome height is less than the circular snap range $R$ in (11) (i.e., two times voxel diagonal length $\delta V$, as listed in Table I. For the intracranial data sets that we have tested, $2 \times \delta V \approx 0.62 \mathrm{~mm}$ ); 2) a fusiform aneurysm whose diameter is less than $2 \times R$ plus the normal diameter of its parent vessel (e.g., in a typical CTA of an abdominal aorta, $\delta V \approx 1.45 \mathrm{~mm}$, then $2 \times R=4 \times \delta V \approx 5.8 \mathrm{~mm}$. The normal diameter of an abdominal aorta is approximately 2 $\mathrm{cm}$ [55]. In other words, our method may have problem with an AAA whose diameter $<2.58 \mathrm{~cm}$, which is 1.29 times the normal diameter of the abdominal aorta). This is because when we deform the explicit vascular surface models to match the boundaries in the segmentations, the algorithm may treat the flat aneurysmal sac and the mild local abnormal arterial dilation as nearby disease-free lumen boundaries and may drive the surfaces marching toward the aneurysmal fundi. In fact these kinds of pathology are not the immediate focus of the project. Although they may not be common (for instance, as shown in Fig. 14(c) and (d), all the dome heights of the intracranial saccular aneurysms measured are $>2 \mathrm{~mm}$, and a segmental dilation of an abdominal aorta with an increase in diameter of less than 1.5 times its normal diameter is not diagnosed as AAA at all [55]), demarcation of such flat sac and mild segmental dilation can be resorted to manual delineation or other methods (if any).

\section{F. Further Possible Applications}

As illustrated in Section V-B, identification of pathological volumes can make the measurements of important clinical parameters become more repeatable. It also helps the angle selection for optimal working projections, and allows interactive or automatic quantitative analyzes on the lesions, which have already been mentioned in Section I. Besides, we see several other possible applications. Upon the pathological structures are recognized from the normal counterparts, study [56], [57] and visualization [58] of hemodynamics on patient specific data sets with/without the pathology of interest prior to endovascular treatments become feasible. Analysis of the normal and abnormal vascular shapes [59], growth simulation of the pathological structures [60], and synthesis of more realistic pathologies with the identified pathological volumes in clinical data sets [61] are also possible. These are all beneficial to endovascular treatment/surgical planning and simulation. In addition, centerlines of the augmented vessels estimated could be used to assist endovascular stent grafting [62] and simulate stent pose [63] in occlusion and recanalization treatments, respectively.

\section{CONCLUSION}

Identification of abnormal vascular structures from a vasculature is beneficial to quantitative analysis of abnormalities, endovascular treatment planning and simulation. It helps 1) to make measurements of clinical parameters more repeatable and effective to take, 2) to allow exclusive quantitative analysis on the lesions, 3) the angle selection for optimal working 
projections, 4) to enable a variety of studies on the normal and abnormal vascular structures, including hemodynamical study and visualization, shape analysis, growth simulation, 5) to synthesize more realistic pathologies for treatment simulation, and 6) to assist/simulate endovascular stent grafting/deployment. Hence, in this paper, we develop a novel unified approach to identifying different vascular abnormalities, for instance, fusiform, narrow- and wide-neck saccular aneurysmal lumens, and stenotic atherosclerotic plaque.

Our method, in contrast to the other algorithms, models the opposite of the abnormalities (i.e., normal vessels) to identify the lesion lumens in an indirect fashion. The normal vessel models, namely augmented vessels, are much easier to manipulate as compared with the model of complex shaped diseased lumens. The abnormal lumens, therefore, can be obtained using a simple set operation. We have tested the method on several synthetic and clinical data sets, and illustrated that it is capable of identifying different kinds of abnormalities as opposed to the two related works, which are restricted to the demarcation of narrow-neck saccular aneurysms only. Furthermore, in an in vivo study with 17 3-D RA cases, we show that our method can help increase the measurement repeatability of clinical parameters by $10+$ times.

A possible improvement to the proposed method is to make it directly applicable to gray-scale images instead of topologically and morphologically correct vascular segmentations. Finally, intensive tests of the method on a clinical environment and studies of its impact on the planning of endovascular treatment, for instance, coil embolization of intracranial aneurysms and recanalization of cerebral arteries, are of interest for future research.

\section{APPENDIX I \\ REFORMULATION OF THE LAGRANGIAN}

The aim of the reformulation is to rewrite the first- and second-order partial derivatives in $E_{\text {tensile }}^{\mathrm{S}}$ and $E_{\text {flexural }}^{\mathrm{S}}$ (see (14) and (15) for the definitions) in terms of the gradient and the Laplacian operators on the parametric space. Hence, the derived motion equations are invariant to the surface parameterization [64], [65]. These energies are reformulated as follows:

$$
\begin{aligned}
E_{\text {tensile }}^{\mathbf{S}} & =\int_{\mathbf{S}}\left|\mathbf{S}_{u}\right|^{2}+\left|\mathbf{S}_{v}\right|^{2} d u d v \\
& =\int_{\mathbf{S}} \sum_{d=1}^{3}\left(\mathbf{S}_{u}^{(d)^{2}}+\mathbf{S}_{v}^{(d)^{2}}\right) d u d v \\
& =\int_{\mathbf{S}} \sum_{d=1}^{3}\left|\nabla \mathbf{S}^{(d)}\right|^{2} d u d v \\
E_{\text {flexural }}^{\mathbf{S}} & =\int_{\mathbf{S}}\left|\mathbf{S}_{u u}\right|^{2}+2\left|\mathbf{S}_{u v}\right|^{2}+\left|\mathbf{S}_{v v}\right|^{2} d u d v \\
& =\int_{\mathbf{S}} \sum_{d=1}^{3}\left(\mathbf{S}_{u u}^{(d)}+2 \mathbf{S}_{u v}^{(d)^{2}}+\mathbf{S}_{v v}^{(d)}\right) d u d v \\
& =\int_{\mathbf{S}} \sum_{d=1}^{3}\left(\left(\nabla^{2} \mathbf{S}^{(d)}\right)^{2}-2 \mathcal{H}\left(\mathbf{S}^{(d)}\right)\right) d u d v
\end{aligned}
$$

where $\mathbf{X}^{(d)}$ represents the $d$ th component of the vector $\mathbf{X}$

$$
\begin{aligned}
\mathcal{H}\left(\mathbf{S}^{(d)}\right) & =\operatorname{det}\left[\begin{array}{ll}
\frac{\partial^{2} \mathbf{S}^{(d)}}{\partial u^{2}} & \frac{\partial^{2} \mathbf{S}^{(d)}}{\partial u \partial v} \\
\frac{\partial^{2} \mathbf{S}^{(d)}}{\partial u \partial v} & \frac{\partial^{2} \mathbf{S}^{(d)}}{\partial v^{2}}
\end{array}\right] \\
& =\mathbf{S}_{u u}^{(d)} \times \mathbf{S}_{v v}^{(d)}-\left(\mathbf{S}_{u v}^{(d)}\right)^{2}
\end{aligned}
$$

is the determinant of the Hessian matrix of $\mathbf{S}^{(d)}$ on the parametric space.

\section{APPENDIX II \\ GRADIENT OF THE EXTERNAL FUNCTIONAL INTEGRAND}

The integrand formulations of the snap and the anti-collapse functionals are very similar. As such, we only derive the gradient of the snap functional integrand. The anti-collapse counterpart could be derived in the same fashion. Consider $\operatorname{ndis} \mathbf{M}(\mathbf{S}) \leq R$ and let $\vec{b}$ denote the vector pointing to $\mathbf{S}(u, v)$ from its corresponding nearest point on $\mathbf{M}$ (i.e., $\vec{b}=\mathbf{S}(u, v)-\mathrm{np}_{\mathbf{M}}(\mathbf{S})$, where $\mathrm{np}_{\mathbf{M}}(\mathbf{S})$ gives the nearest point on $\mathbf{M}$ from $\mathbf{S}(u, v)$ ). Then we have the following equations:

$$
\begin{aligned}
\operatorname{ndis}_{\mathbf{M}}(\mathbf{S}) & =|\vec{b}|=\sqrt{\sum_{d=1}^{3}\left(b^{(d)}\right)^{2}} \\
\mathcal{E}_{\text {snap }}^{\mathbf{S}} & =\operatorname{ndisM}(\mathbf{S})+p \times e^{\frac{-\operatorname{ndis}_{\mathbf{M}}(\mathbf{S})}{p}} \\
& =|\vec{b}|+p \times e^{\frac{-|\vec{b}|}{p}} \\
\frac{\partial}{\partial x} \mathcal{E}_{\text {snap }}^{\mathbf{S}} & =\frac{b^{(1)}}{|\vec{b}|}-e^{\frac{-|\vec{b}|}{p}} \times \frac{b^{(1)}}{|\vec{b}|} \\
& =\left(1-e^{\frac{-|\vec{b}|}{p}}\right) \times \frac{b^{(1)}}{|\vec{b}|}
\end{aligned}
$$

therefore, the gradient of the integrand is given as

$$
\nabla \mathcal{E}_{\text {snap }}^{\mathrm{S}}=\left(1-e^{\frac{-|\vec{b}|}{p}}\right) \times \frac{\vec{b}}{|\vec{b}|}
$$

when ndism $(\mathbf{S}) \leq R$. However, we also want this gradient vanishes if $\operatorname{ndis}_{\mathbf{M}}(\mathbf{S})>R$ and is not undefined when $\vec{b}=\overrightarrow{0}$. Thus, we replace the numerator of the multiplicand in $\nabla \mathcal{E}_{\text {snap }}^{\mathbf{S}}$ with $\overrightarrow{0}$ when its magnitude is greater than $R$ and set a nonzero lower bound for the denominator such that the integrand gradient is well-defined. The gradient is, therefore, expressed as

$$
\nabla \mathcal{E}_{\text {snap }}^{\mathbf{S}}=\left(1-e^{\frac{-\mathrm{ndis}_{\mathbf{M}}(\mathbf{S})}{p}}\right) \times \frac{\mathbf{S}-\mathrm{np}_{\mathbf{M}}^{*}(\mathbf{S})}{\operatorname{ndis}_{\mathbf{M}}^{*}(\mathbf{S})}
$$

where

$$
\operatorname{np}_{\mathbf{M}}^{*}(\mathbf{S})= \begin{cases}\operatorname{np}_{\mathbf{M}}(\mathbf{S}), & \operatorname{ndis}_{\mathbf{M}}(\mathbf{S}) \leq R \\ \mathbf{S}, & \text { otherwise }\end{cases}
$$

and

$$
\operatorname{ndis}_{\mathbf{M}}^{*}(\mathbf{S})=\max \left(\epsilon, \operatorname{ndis}_{\mathbf{M}}(\mathbf{S})\right)
$$

$\mathbf{S}$ is an abbreviation for $\mathbf{S}(u, v)$ and the constant $\epsilon$ denotes a very small positive real number. 


\section{ACKNOWLEDGMENT}

The authors wish to thank Dr. S. C. H. Yu of the Department of Diagnostic Radiology and Organ Imaging, Prince of Wales Hospital, Hong Kong, for providing the clinical data and the fruitful discussions, in particular, the idea of deploying virtually a stent at a coarctation for treatment planning, which inspires the initial development of this paper; Mr. T. W. H. Tang for performing the measurements of the aneurysmal neck widths and dome heights on the tested data sets. They would also like to thank the anonymous reviewers for their perceptive comments, which have significantly improved the paper.

\section{REFERENCES}

[1] W. S. Moore and S. S. Ahn, Endovascular Surgery, 3rd ed. Philadelphia, PA: Saunders, 2001

[2] G. J. Rinkel, M. Djibuti, A. Algra, and J. van Gijn, "Prevalence and risk of rupture of intracranial aneurysms: a systematic review," Stroke, vol. 29, pp. 251-256, 1998.

[3] T. Abe, M. Hirohata, N. Tanaka, Y. Uchiyama, K. Kojima, K. Fujimoto, A. M. Norbash, and N. Hayabuchi, "Clinical benefits of rotational 3D angiography in endovascular treatment of ruptured cerebral aneurysm," Am. J. Neuroradiol., vol. 23, pp. 686-688, 2002.

[4] W. P. Sanders, T. H. Burke, and B. A. Mehta, "Embolization of intracranial aneurysms with Guglielmi detachable coils augmented by microballoons," Am. J. Neuroradiol., vol. 19, pp. 917-920, 1998.

[5] F. Turjman, T. F. Massoud, J. Sayre, and F. Viñuela, "Predictors of aneurysmal occlusion in the period immediately after endovascular treatment with detachable coils: a multivariate analysis," Am. J. Neuroradiol., vol. 19, pp. 1645-1651, 1998.

[6] S. M. Pizer, P. T. Fletcher, S. Joshi, A. Thall, J. Z. Chen, Y. Fridman, D. S. Fritsch, A. G. Gash, J. M. Glotzer, M. R. Jiroutek, C. Lu, K. E. Muller, G. Tracton, P. Yushkevich, and E. L. Chaney, "Deformable m-reps for 3D medical image segmentation," Int. J. Comput. Vis., vol. 55, no. 2-3, pp. 85-106, Nov. 2003.

[7] Y. Sato, T. Araki, M. Hanayama, H. Naito, and S. Tamura, "A viewpoint determination system for stenosis diagnosis and quantification in coronary angiographic image acquisition," IEEE Trans. Med. Imag., vol. 17 , no. 1, pp. 121-137, Feb. 1998.

[8] R. van der Weide, K. J. Zuiderveld, W. P. T. M. Mali, and M. A. Viergever, "CTA-based angle selection for diagnostic and interventional angiography of saccular intracranial aneurysms," IEEE Trans. Med. Imag., vol. 17, no. 5, pp. 831-841, Oct. 1998.

[9] D. L. Wilson, D. D. Royston, J. A. Noble, and J. V. Byrne, "Determining X-ray projections for coil treatments of iniracranial aneurysms," IEEE Trans. Med. Imag., vol. 18, no. 10, pp. 973-980, Oct. 1999.

[10] R. A. McLaughlin and J. A. Noble, "Demarcation of aneurysms using the seed and cull algorithm," in Lecture Notes in Comput. Science. Berlin, Germany: Springer-Verlag, 2002, vol. 2488, Medical Image Computing and Computer Assisted Intervention, pp. 419-426.

[11] J. Vallino, "Interactive augmented reality," Ph.D. dissertation, Dept. Comput. Sci., Univ. Rochester, Rochester, NY, Apr. 1998.

[12] M. Egmont-Petersen, D. de Ridder, and H. Handels, Vascular and Interventionat Radiology: Principles and Practice. : Thieme Medical Publishers, Inc., 2002.

[13] A. F. Frangi, W. J. Niessen, R. M. Hoogeveen, T. V. Walsum, and M. A. Viergever, "Model-based quantitation of 3-D magnetic resonance angiographic images," IEEE Trans. Med. Imag., vol. 18, no. 10, pp. 946-956, Oct. 1999.

[14] P. J. Yim, R. Mullick, R. M. Summers, H. Marcos, J. R. Cebral, R. Löhner, and P. L. Choyke, "Measurement of stenosis from magnetic resonance angiography using vessel skeletons," Proc. SPIE (Med. Imag.), vol. 3978, pp. 245-255, 2000.

[15] E. Bullitt, S. Aylward, K. Smith, S. Mukherji, M. Jiroutek, and K. Muller, "Symbolic description of intracerebral vessels segmented from magnetic resonance angiograms and evaluation by comparison with X-ray angiograms," Med. Imag. Anal., vol. 5, pp. 157-169, 2001.

[16] P. J. Yim, J. J. Cebral, R. Mullick, H. B. Marcos, and P. L. Choyke, "Vessel surface reconstruction with a tubular deformable model," IEEE Trans. Med. Imag., vol. 20, no. 12, pp. 1411-1421, Dec. 2001.
[17] S.R. Aylward and E. Bullitt, "Initialization, noise, singularities, and scale in height ridge traversal for tubular object centerline exiraction," IEEE Trans. Med. Imag., vol. 21, no. 2, pp. 61-75, Feb. 2002.

[18] O. Wink, W. J. Niessen, and M. A. Viergever, "Multiscale vessel tracking," IEEE Trans. Med. Imag., vol. 23, no. 1, pp. 130-133, Jan. 2004.

[19] H. C. Schumacher, K. Tanji, S. Mangla, P. Meyers, J. Pile-Spellman, A. P. Hays, and J. P. Mohr, "Histopathological evaluation of middle cerebral artery after percutaneous intracranial transluminal angioptasty," Stroke, vol. 34, no. 9, pp. 170-173, 2003.

[20] J. Jomier and S. R. Aylward, "Rigid and deformable vasculature-to-image registration: a hierarchical approach," in Lecture Notes in Comput. Science. Berlin, Germany: Springer-Verlag, 2004, vol. 3216, Medical Image Computing and Computer Assisted Intervention, pp. 829-836.

[21] M. Kass, A. Witkin, and D. Terzopoulos, "Snakes: active contour models," Int. J. Comput. Vision, vol. 1, pp. 321-331, 1988.

[22] O. Wink, W. J. Niessen, and M. A. Viergever, "Fast delineation and visualization of vessels in 3-D angiographic images," IEEE Trans. Med. Imag., vol. 19, no. 4, pp. 337-346, Apr. 2000.

[23] S. R. Aylward, J. Jomier, S. Weeks, and E. Bullitt, "Registration and analysis of vascular images," Int. J. Comput. Vision, vol. 55, no. 2-3, pp. 123-138, Nov. 2003

[24] D. Tang, C. Yang, S. Kobayashi, J. Zheng, and R. P. Vito, "Effect of stenosis asymmetry on blood flow and artery compression: a three-dimensional fluid-structure interaction model," Ann. Biomed. Eng., vol. 31, pp. 1182-1193, 2003.

[25] D. Rueckert, L. I. Sonoda, C. Hayes, D. L. G. Hill, M. O. Leach, and D. J. Hawkes, "Nonrigid registration using free-form deformations: application to breast MR images," IEEE Trans. Med. Imag., vol. 18, no. 8 , pp. 712-721, Aug. 1999.

[26] K. L. Moore and A. F. Dalley, Clinically Oriented Anatomy, 4th ed. Baltimore, MD: Lippincott Williams \& Wilkins, 1999.

[27] W. E. Lorensen and H. E. Cline, "Marching cubes: a high resolution 3D surface construction algorithm," Comput. Graph., vol. 21, no. 4, pp. 163-169, 1987

[28] V. Caselles, R. Kimmel, and G. Sapiro, "Geodesic active contours," Int. J. Comput. Vis., vol. 22, no. 1, pp. 61-79, 1997.

[29] C. Xu and J. L. Prince, "Snakes, shapes, and gradient vector flow," IEEE Trans. Image Process., vol. 7, no. 3, pp. 359-369, Mar. 1998.

[30] T. McInerney and D. Terzopoulos, "Topology adaptive deformable surfaces for medical image volume segmentation," IEEE Trans. Med. Imag., vol. 18, no. 10, pp. 840-850, Oct. 1999.

[31] G. Giraldi, E. Strauss, and A. Oliveira, "Dual-T-Snakes model for medical imaging segmentation," Pattern Recognit. Lett., vol. 24, no. 7, pp. 993-1003, 2003.

[32] T. McInemey and D. Terzopoulos, "T-snakes: topology adaptive snakes," Med. Imag. Anal., vol. 4, no. 2, pp. 73-91, 2000.

[33] Kitware, Inc., 2005, The Insight Segmentation and Registration Toolkit ITK [Online]. Available: http://www.itk.org

[34] Kitware, Inc., 2005, The Visualization ToolKit VTK [Online]. Available: http://www.vtk.org

[35] J. Smart et al., 2005, wxWidgets [Online]. Available: http://www. wxwidgets.org

[36] Y. Zhou and A. W. Toga, "Efficient skeletonization of volumetric objects," IEEE Trans. Visual. Comput. Graphics, vol. 5, no. 3, pp. 196-209, Jul.-Sep. 1999.

[37] T. Saito and J. I. Toriwaki, "New algorithms for Euclidean distance transformations of an n-dimensional digitized picture with applications," Pattern Recognit., vol. 27, no. 11, pp. 1551-1565, 1994.

[38] M. Styner, G. Gerig, C. Brechbuehler, and G. Szekely, "Parametric estimate of intensity inhomogeneities applied to MRI," IEEE Trans. Med. Imag., vol. 19, no. 3, pp. 153-165, Mar. 2000.

[39] R. L. Kashyap and A. Khotanzad, "A model-based method for rotation invariant texture classification," IEEE Trans. Pattern Anal. Machine Intell., vol. PAMI-8, no. 4, pp. 472-481, Apr. 1986.

[40] J. D. Hoffman, Numerical Methods for Engineers and Scientists, 2nd ed. New York: Marcel Dekker, Sept. 2001.

[41] W. J. Schrder, J. A. Zarge, and W. E. Lorensen, "Decimation of triangle meshes," Comput. Graph., vol. 26, no. 2, pp. 65-70, July 1992.

[42] H. Hoppe, "New quadric metric for simplifying meshes with appearance attributes," Visualization, pp. 59-66, 1999.

[43] A. Criminisi, P. Pérez, and K. Toyama, "Region filling and object removal by exemplar-based image inpainting," IEEE Trans. Image Process., vol. 13, no. 9, pp. 1200-1212, Sep. 2004.

[44] J. V. Byrne and G. Guglielmi, Endovascular Treatment of Intracranial Aneurysms. Berlin, Germany: Springer-Verlag, 1997. 
[45] R. O. Duda, P. E. Hart, and D. G. Stork, Pattern Classification, 2nd ed. New York: Wiley Interscience, 2000.

[46] R. V. Hogg and J. Ledolter, Applied Statistics for Engineers and Physical Scientists, R. W. Pirtle, Ed., 2nd ed. Toronto, ON, Canada: Maxwell Macmillan, 1992.

[47] L. Parlea, R. Fahrig, D. W. Holdsworth, and S. P. Lownie, "An analysis of the geometry of saccular intracranial aneurysms," Am. J. Neuroradiol., vol. 20, pp. 1079-1089, June 1999.

[48] J. M. Bland and D. G. Altman, "Statistical methods for assessing agreement between two methods of clinical measurement," Lancet, vol. 1, pp. 307-310, 1986.

[49] NASCET, "North American symptomatic carotid endarterectomy trial," Stroke, vol. 22, pp. 711-720, 1991.

[50] S. M. Pizer, D. Eberly, D. S. Fritsch, and B. S. Morse, "Zoom-invariant vision of figural shape: the mathematics of cores," Comput. Vis. Image Understanding, vol. 69, no. 1, pp. 55-71, Jan. 1998.

[51] J. D. Furst, S. M. Pizer, and D. H. Eberly, "Marching cores: a method for extracting cores from 3D medical images," in Proc. Workshop Mathematical Methods in Biomedical Image Analysis, 1996, pp. $124-130$.

[52] Y. Fridman, S. M. Pizer, S. Aylward, and E. Bullitt, "Segmenting 3D branching tubular structures using cores," in Lecture Notes in Comput. Science. Berlin, Germany: Springer-Verlag, Jan. 2003, vol. 2879, Medical Image Computing and Computer Assisted Intervention, pp. 570-577.

[53] E. Bullitt, K. E. Muller, I. Jung, W. Lin, and S. Aylward, "Analyzing attributes of vessel populations," Med. Imag. Anal., vol. 9, pp. 39-49, 2004.

[54] D. F. Schomer, M. P. Marks, G. K. Steinberg, I. M. Johnstone, D. B. Boothroyd, M. R. Ross, N. J. Pelc, and D. R. Enzmann, "The anatomy of the posterior communicating artery as a risk factor for ischemic cerebral infarction," New Engl. J. Med., vol. 330, no. 22, pp. 1565-1570, Jun. 1994.

[55] J. D. Santilli and S. M. Santilli, "Diagnosis and treatment of abdominal aortic aneurysms," Am. Fam. Physician, vol. 56, no. 4, pp. 1081-1090, Sep. 1997.
[56] T. Hassan, M. Ezura, E. V. Timofeev, T. Tominaga, T. Saito, A. Takahashi, K. Takayama, and T. Yoshimoto, "Computational simulation of therapeutic parent artery occlusion to treat giant vertebrobasilar aneurysm," Am. J. Neuroradiol., vol. 25, pp. 63-68, Jan. 2004.

[57] J. R. Cebral, M. A. Castro, S. Appanaboyina, C. M. Putman, D. Millan, and A. F. Frangi, "Efficient pipeline for image-based patient-specific analysis of cerebral aneurysm hemodynamics: technique and sensitivity," IEEE Trans. Med. Imag., vol. 24, no. 4, pp. 457-467, Apr. 2005.

[58] M. D. Ford, G. R. Stuhne, H. N. Nikolov, D. F. Habets, S. P. Lownie, D. W. Holdsworth, and D. A. Steinman, "Virtual angiography for visualization and validation of computational models of aneurysm hemodynamics," IEEE Trans. Med. Imag., vol. 24, no. 12, pp. 1586-1592, Dec. 2005

[59] D. Selle, B. Preim, A. Schenk, and H.-O. Peitgen, "Analysis of vasculature for liver surgical planning," IEEE Trans. Med. Imag., vol. 21, no. 11, pp. 1344-1357, Nov. 2002.

[60] D. Szczerba and G. Székely, "Simulating vascular systems in arbitrary anatomies," in Lecture Notes in Comput. Science. Berlin: SpringerVerlag, 2005, vol. 3750, Medical Image Computing and Computer Assisted Intervention, pp. 641-648.

[61] T. Alderliesten, K. M. Konings, and W. J. Niessen, "Simulation of minimally invasive vascular interventions for training purposes," Comput. Aided Surg., vol. 9, no. 1-2, pp. 3-15, 2004.

[62] S. Eiho, H. Imamura, and N. Sugimoto, "Preoperative and intraoperative image processing for assisting endovascular stent grafting," in Proc. Int. Conf. Informatics Research for Development of Knowledge Society Infrastructure (ICKS'04) , 2004, pp. 81-88.

[63] L. Flórez-Valencia, J. Montagnat, and M. Orkisz, "3D graphical models for vascular-stent pose simulation," in Int. Conf. Computation, Vision, and Graph., Sept. 2002.

[64] W. Neuenschwander, P. Fua, G. Székely, and O. Kübler, "Deformable velcro surfaces," in Int. Conf. Computer Vision, 1995, pp. 828-833.

[65] W. Neuenschwander, "Elastic deformable contour and surface models for 2-D and 3-D image segmentation," Ph.D. dissertation, Swiss Federal Inst. Technol., Zurich, Switzerland, 1995. 